Article

\title{
Accelerated Life Tests under Pareto-IV Lifetime Distribution: Real Data Application and Simulation Study
}

\author{
A. M. Abd El-Raheem ${ }^{1}\left(\mathbb{D}\right.$, M. H. Abu-Moussa $^{2}{ }^{(D}$, Marwa M. Mohie El-Din ${ }^{3}(\mathbb{D}$ \\ and E. H. Hafez $4, *$ (D) \\ 1 Department of Mathematics, Faculty of Education, Ain Shams University, Cairo 11566, Egypt; \\ abdelrahimmohamed@edu.asu.edu.eg \\ 2 Department of Mathematics, Faculty of Science, Cairo University, Giza 12511, Egypt; \\ mhmoussa@sci.cu.edu.eg \\ 3 Department of Mathematics, Faculty of Engineering, Egyptian Russian University, Cairo 11829, Egypt; \\ marwa-mostafa@eru.edu.eg \\ 4 Department of Mathematics, Faculty of Science, Helwan University, Cairo 11795, Egypt \\ * Correspondence: eslamhussam@science.helwan.edu.eg; Tel.: +20-1094506495
}

Received: 7 September 2020; Accepted: 4 October 2020 ; Published: 15 October 2020

\begin{abstract}
In this article, a progressive-stress accelerated life test (ALT) that is based on progressive type-II censoring is studied. The cumulative exposure model is used when the lifetime of test units follows Pareto-IV distribution. Different estimates as the maximum likelihood estimates (MLEs) and Bayes estimates (BEs) for the model parameters are discussed. Bayesian estimates are derived while using the Tierney and Kadane (TK) approximation method and the importance sampling method. The asymptotic and bootstrap confidence intervals (CIs) of the parameters are constructed. A real data set is analyzed in order to clarify the methods proposed through this paper. Two types of the progressive-stress tests, the simple ramp-stress test and multiple ramp-stress test, are compared through the simulation study. Finally, some interesting conclusions are drawn.
\end{abstract}

Keywords: progressive-stress; progressive type-II censoring; maximum likelihood estimation; bayes estimation; Tierney and Kadane approximation; simulation study

\section{Introduction}

In most of the classical life testing and reliability experiments, collecting enough number of failure times is not easy, especially when the products are highly reliable with long lifetimes. Under normal conditions the lifetime experiments with a restricted testing time may produce a very few failures, one of the most famous procedures used to accelerate the occurrence of failure is the ALT, in which products are tested at high levels of stress (e.g., humidity, temperature, pressure, voltage, or vibration). The failure time data from ALTs are then analyzed in order to estimate the life characteristics of the products under normal conditions. There are different ways in which the ALT can work, for example, constant, step, and progressive stress ALT. Nelson discussed these different types [1].

In constant-stress ALT, the test is performed completely under a constant level of stress. Several authors have discussed the constant-stress models; see references [2-9] for more details. The second way to apply accelerated stress is step-stress. In this kind of stress, we raise the stress level step by step at pre-specified times to get early failures. Many authors have discussed the step-stress ALTs; see for example Bai et al. [10], Gouno et al. [11], Miller and Nelson [12], Balakrishnan et al. [13], and Mohie El-Din et al. [14-17]. On the other hand, in progressive-stress ALT, all of the test units are exposed to stress, which is continuously at an increasing rate with time. For more reading 
about progressive-stress ALT, see Yin and Sheng [18], Mohie El-Din et al. [19], Bai et al. [20], as well as Ronghua and Heliang [21] and Abdel-Hamid and AL-Hussaini [22] who also studied the progressive-stress ALT based on progressive censoring in case of Weibull distribution in [23]. Bayesian prediction intervals under progressively type-II censoring for the half-logistic distribution under progressive-stress model have been discussed by AL-Hussaini et al. [24]. The Bayesian estimates of exponentiated exponential distribution based on type-II progressive hybrid censoring have been discussed by Abdel-Hamid and Abushul [25], depending on the inverse power law and the cumulative exposure model. Abd El-Raheem [26] considered the problem of optimal design of progressive-stress ALT for generalized half normal distribution.

Because of the high cost and long time that the life testing experiments consume, the experimenter needs to end the experiment before observing all failures, so the censoring techniques of data are widely used in order to decrease the test time and cost. Type-I and Type-II conventional censoring are the most two used censoring schemes for life testing. Recently, the most popular censoring type is the progressive type-II censoring scheme (CS). It can be illustrated, as follows: suppose $n$ identical units or devices are put on a life time test with $m \leq n$ is a pre-specified number of failures. At the occurrence of the first failure $t_{1: m: n}, R_{1}$ surviving units are removed from the test. When the second failure $t_{2: m: n}$ occurs, $R_{2}$ surviving units are also removed from the test and so on until the $m$-th failure is observed, where the test is terminated and the remaining surviving $R_{m}=n-m-\left(R_{1}+\ldots+R_{m-1}\right)$ units are removed. For extra studies regarding progressive censoring, see [27,28].

The contribution in this paper is studying statistical inference of progressive-stress ALT for test units whose lifetime follow Pareto-IV distribution in the presence of progressively type-II censored data.

The paper is organized, as follows: in Section 2, the ALT model and test assumptions are presented. In Section 3, the maximum likelihood estimates (MLEs) of the parameters under progressive-stress model are obtained. Using Tierney and Kadane (TK) Approximation and importance sampling method, the Bayes estimates (BEs) for model parameters are discussed in Section 4. The asymptotic and bootstrap confidence intervals for the parameters of the model are constructed in Section 5 . In Section 6, a real data set is analyzed in order to illustrate the proposed methods. Section 7 includes the simulation study. Finally, the paper is concluded in Section 8.

\section{ALT Model and Test Assumptions}

\subsection{Pareto-IV Distribution}

Arnold presents the hierarchy of Pareto-IV distribution [29]. It was formed by adding additional parameters (shape, scale, location, and inequality) to the conventional Pareto I distribution. The cumulative distribution function (CDF) of Pareto-IV $(\mu, \gamma, \theta, \alpha)$ distribution is given by

$$
F(t)=1-\left[1+\left(\frac{t-\mu}{\theta}\right)^{1 / \gamma}\right]^{-\alpha}, \quad \gamma, \alpha, \theta>0, t>\mu,-\infty<\mu<\infty .
$$

With a view to present Pareto-IV distribution as a regular family, we consider parameter $\mu$ is known and equal to 0 , Serfling [30]. Thus, the corresponding probability density function (PDF) of Pareto-IV $(0, \gamma, \theta, \alpha)$ is given by

$$
f(t)=\frac{\alpha\left(\frac{t}{\theta}\right)^{\frac{1}{\gamma}-1}}{\gamma \theta\left(1+\left(\frac{t}{\theta}\right)^{\frac{1}{\gamma}}\right)^{\alpha+1}} \quad, t>0, \gamma, \alpha, \theta>0 .
$$




\subsection{Test Assumptions}

In this subsection, we list the assumptions that are used through this article in the context of progressive-stress ALT:

1. The lifetime of a unit follows Pareto-IV $(0, \gamma, \theta, \alpha)$.

2. The progressive-stress at level $i, S_{i}(t)$ is directly proportional to the time with fixed rate $v_{i}$, i.e., $S_{i}(t)=v_{i} t, 0<v_{1}<v_{2}<\ldots<v_{k}$.

3. The inverse power law is used to model the relationship between the life characteristic $\theta_{i}$ and the stress loading $S_{i}(t)$, as follows

$$
\theta_{i}(t)=\frac{1}{a\left[s_{i}(t)\right]^{b}},
$$

where the parameters $a$ and $b$ are unknown and positive. For more information about this acceleration model, see, e.g., Nelson ([1], [Ch. 2]).

4. The cumulative exposure model is used in order to describe the effect of stress on failure time from one level to another, see, e.g., Nelson [1].

5. From the life-stress relationship in (3) the parameter $\theta_{i}$ can be expressed as $\theta_{i}=\theta_{1} \psi_{i}^{b}$, where $\psi_{i}=\frac{v_{1}}{v_{i}}$.

Suppose that the test is started with $n$ units, $S_{0}<S_{1}(t)<\ldots<S_{k}(t)$ be the stress levels used during the test and $S_{0}$ be the use-stress. Furthermore, suppose $n_{i}$ units are tested under each progressive-stress level $S i(t), i=1,2, \ldots, k$. The progressive censoring scheme is carried out as follows: When the time of the first failure $t_{i 1: m_{i}: n_{i}}$ is reached, $R_{i 1}$ units are randomly removed from the remaining $n_{i}-1$ surviving units. When the time of the second failure $t_{i 2: m_{i}: n_{i}}$ is reached, $R_{i 2}$ units from the remaining $n_{i}-2-R_{i 1}$ surviving units are randomly withdrawn from the test. The test is terminated at level $i$ when the $m_{i}$-th failure occurs $t_{i m_{i}: m_{i}: n_{i}}$, at this time all remaining $R_{i m_{i}}=n_{i}-m_{i}-\sum_{j=1}^{m_{i}-1} R_{i j}$ items are withdrawn. It is clear that the complete samples and type-II censored samples are special cases of this scheme. The observed life times while using the progressive-stress $S_{i}(t)$ are $t_{i 1: m_{i}: n_{i}}<t_{i 2: m_{i}: n_{i}}<\ldots<$ $t_{i m_{i}: m_{i}: n_{i}}, i=1,2, \ldots, k$.

Based on the assumption of the of cumulative exposure model, the CDF for the lifetimes that result from progressive-stress $S_{i}(t)$ is

$$
G_{i}(t)=F_{i}(\triangle(t)), i=1,2, \ldots, k,
$$

where $\triangle(t)=\int_{0}^{t} \frac{d u}{\theta_{i}(u)}=\frac{t}{\theta_{1} \psi_{i}^{b}(b+1)}$, and $F_{i}($.$) is defined in (2) with scale parameter equals 1$. Therefore,

$$
G_{i}(t)=1-\left[1+\left(\frac{t}{\sigma i}\right)^{1 / \gamma}\right]^{-\alpha}, t>0, \gamma, \alpha, \sigma i>0,
$$

where $\sigma i=\theta_{1} \psi_{i}^{b}(b+1)$. The PDF of (5) is given by

$$
g_{i}(t)=\frac{\alpha\left(\frac{t}{\sigma i}\right)^{\frac{1}{\gamma}-1}}{\gamma \sigma i\left(1+\left(\frac{t}{\sigma i}\right)^{\frac{1}{\gamma}}\right)^{\alpha+1}}, t>0, \gamma, \alpha, \sigma i, \theta>0
$$

\section{Maximum Likelihood Estimation}

In this section, the MLEs of the parameters $\gamma, \alpha, \theta 1$, and $b$ are obtained. For simplification, let $t_{i j: m_{i}: n_{i}}=t_{i j}$, which represent the progressive censoring lifetimes under progressive-stress level $S_{i}(t), i=1,2, \ldots, k$ and $j=1,2, \ldots, m_{i}$. The likelihood function of the proposed model is derived while using the CDF in (5) and the corresponding PDF in (6), as follows:

$$
L\left(\gamma, \alpha, \theta_{1}, b\right)=\prod_{i=1}^{k} C_{i} \prod_{j=1}^{m_{i}} g_{i}\left(t_{i j}\right)\left[1-G_{i}\left(t_{i j}\right)\right]^{R_{i j}},
$$


where $C_{i}=n_{i}\left(n_{i}-1-R_{i 1}\right)\left(n_{i}-2-R_{i 1}-R_{i 2}\right) \ldots\left(n_{i}-m_{i}+1-\sum_{j=1}^{m_{i}-1} R_{i j}\right)$.

From (5) and (6) in (7), we obtain

$$
L\left(\gamma, \alpha, \theta_{1}, b\right)=\prod_{i=1}^{k} C_{i} \prod_{j=1}^{m_{i}} \frac{\alpha\left(\frac{t_{i j}}{\sigma i}\right)^{\frac{1}{\gamma}-1}}{\gamma \sigma i\left(1+\left(\frac{t_{i j}}{\sigma i}\right)^{\frac{1}{\gamma}}\right)^{\alpha\left(R_{i j}+1\right)+1}} .
$$

Therefore, the log-likelihood function is given by

$$
\begin{aligned}
\ell\left(\gamma, \alpha, \theta_{1}, b\right) & =\sum_{i=1}^{k} \log C_{i}+\left(\log \alpha-\log \sigma_{i}-\log \gamma\right) \sum_{i=1}^{k} m_{i}+\left(\frac{1}{\gamma}-1\right) \sum_{i=1}^{k} \sum_{j=1}^{m_{i}} \log \left(\frac{t_{i j}}{\sigma i}\right) \\
& -\sum_{i=1}^{k} \sum_{j=1}^{m_{i}}\left(\alpha\left(R_{i j}+1\right)+1\right) \log \left(\left(\frac{t_{i j}}{\sigma i}\right)^{\frac{1}{\gamma}}+1\right),
\end{aligned}
$$

where the likelihood equations of $\alpha, \gamma$, and $\theta_{1}$ and $b$ are, respectively

$$
\begin{gathered}
\frac{\partial \ell}{\partial \alpha}=\frac{\sum_{i=1}^{k} m_{i}}{\alpha}-\sum_{i=1}^{k} \sum_{j=1}^{m_{i}}\left[\left(R_{i j}+1\right) \log \left(\left(\frac{t_{i j}}{\sigma i}\right)^{\frac{1}{\gamma}}+1\right)\right], \\
\frac{\partial \ell}{\partial \gamma}=\frac{-\sum_{i=1}^{k} m_{i}}{\gamma}+\sum_{i=1}^{k} \sum_{j=1}^{m_{i}} \frac{-1}{\gamma^{2}} \log \left(\frac{t_{i j}}{\sigma i}\right)+\sum_{i=1}^{k} \sum_{j=1}^{m_{i}} \frac{\left(\alpha\left(R_{i j}+1\right)+1\right)\left(\frac{t_{i j}}{\sigma i}\right)^{\frac{1}{\gamma}} \log \left(\frac{t_{i j}}{\sigma i}\right)}{\gamma^{2}\left(\left(\frac{t_{i j}}{\sigma i}\right)^{\frac{1}{\gamma}}+1\right)}, \\
\frac{\partial \ell}{\partial \theta_{1}}=\frac{-\sum_{i=1}^{k} m_{i}}{\theta_{1}}-\left(\frac{1}{\gamma}-1\right) \sum_{i=1}^{k} \sum_{j=1}^{m_{i}} \frac{1}{\theta_{1}}+\sum_{i=1}^{k} \sum_{j=1}^{m_{i}} \frac{\left(\alpha\left(R_{i j}+1\right)+1\right)\left(\frac{t_{i j}}{\sigma i}\right)^{\frac{1}{\gamma}}}{\gamma \theta_{1}\left(\left(\frac{t_{i j}}{\sigma i}\right)^{\frac{1}{\gamma}}+1\right)} \\
\frac{\partial \ell}{\partial b}=\sum_{i=1}^{k} \sum_{j=1}^{m_{i}}\left[\frac{-1}{(b+1)}-\log \psi\right]+\sum_{i=1}^{k} \sum_{j=1}^{m_{i}}\left(\frac{1}{\gamma}-1\right)\left[\frac{-1}{(b+1)}-\log \psi\right] \\
+\sum_{i=1}^{k} \sum_{j=1}^{m_{i}} \frac{\left(\alpha\left(R_{i j}+1\right)+1\right)\left[(b+1) \log \psi_{i}+1\right]\left(\frac{t_{i j}}{\sigma i}\right)^{\frac{1}{\gamma}}}{\gamma(b+1)\left(\left(\frac{t_{i j}}{\sigma i}\right)^{\frac{1}{\gamma}}+1\right)}
\end{gathered}
$$

The MLEs of the parameters are obtained by solving the system of four nonlinear Equations (10)-(13) in four unknowns $\gamma, \alpha, \theta_{1}$, and $b$. This can be solved while using the Newton-Raphson iteration method.

\section{Bayesian Estimation}

In this section, based on square error (SE), linear exponential (LINEX), and the general entropy (GE) loss functions, the BEs for the parameters $\alpha, \gamma, \theta_{1}$, and $b$ based on progressive type-II censoring are discussed. The prior distributions of the parameters $\alpha, \gamma, \theta_{1}$, and $b$ are assumed to be gamma priors. Thus,

$$
\begin{array}{ll}
\pi_{1}(\alpha) \propto \alpha^{\mu_{1}-1} e^{\frac{-\alpha}{\lambda_{1}}}, & \alpha>0, \mu_{1}, \lambda_{1}>0, \\
\pi_{2}\left(\theta_{1}\right) \propto \theta_{1}^{\mu_{2}-1} e^{\frac{-\theta_{1}}{\lambda_{2}}}, & \theta_{1}>0, \mu_{2}, \lambda_{2}>0, \\
\pi_{3}(b) \propto b^{\mu_{3}-1} e^{\frac{-b}{\lambda_{3}}}, & b>0, \mu_{3}, \lambda_{3}>0,
\end{array}
$$


and

$$
\pi_{4}(\gamma) \propto \gamma^{\mu_{4}-1} e^{\frac{-\gamma}{\lambda_{4}}}, \quad \gamma>0, \mu_{4}, \lambda_{4}>0
$$

Assume that the parameters of the model are independent, then the joint PDF of prior is given by

$$
\pi\left(\gamma, \alpha, \theta_{1}, b\right) \propto b^{\mu_{3}-1} \theta_{1}^{\mu_{2}-1} \alpha^{\mu_{1}-1} \gamma^{\mu_{4}-1} e^{-\left(\frac{\alpha}{\lambda_{1}}+\frac{\gamma}{\lambda_{4}}+\frac{\theta_{1}}{\lambda_{2}}+\frac{b}{\lambda_{3}}\right)} .
$$

The posterior density function of the parameters $\gamma, \alpha, \theta_{1}$ and $b$ can be obtained from (8) and (14), as follows:

$$
\begin{aligned}
\pi^{*}(\Theta \mid t)=\pi^{*}\left(\gamma, \alpha, \theta_{1}, b \mid t\right) & \propto L\left(\gamma, \alpha, \theta_{1}, b\right) \pi\left(\gamma, \alpha, \theta_{1}, b\right) \\
& \propto b^{\mu_{3}-1} \theta_{1}^{\mu_{2}-1} \alpha^{\mu_{1}-1} \gamma^{\mu_{4}-1} e^{-\left(\frac{\alpha}{\lambda_{1}}+\frac{\gamma}{\lambda_{4}}+\frac{\theta_{1}}{\lambda_{2}}+\frac{b}{\lambda_{3}}\right)} \\
& \prod_{i=1}^{k} C_{i} \prod_{j=1}^{m_{i}} \frac{\alpha\left(\frac{t_{i j}}{\sigma i}\right)^{\frac{1}{\gamma}-1}}{\gamma \sigma i\left(1+\left(\frac{t_{i j}}{\sigma i}\right)^{\frac{1}{\gamma}}\right)^{\alpha\left(R_{i j}+1\right)+1}} .
\end{aligned}
$$

The BE of the function of parameters $U=U(\Theta), \Theta=\left(\gamma, \alpha, \theta_{1}, b\right)$ under the SE loss function is given by

$$
\hat{U}_{S E}=\int_{\Theta} U \pi^{*}(\Theta \mid t) d \Theta,
$$

Depending on the LINEX loss function, the BE of $U=U(\Theta)$ is given by

$$
\hat{U}_{\text {LINEX }}=-\frac{1}{c} \log \left[\int_{\Theta} e^{-c U} \pi^{*}(\Theta \mid t) d \Theta\right],
$$

where $c \neq 0$ is the shape parameter of the LINEX loss function.

Based on GE loss function, the BE of $U$ is given by

$$
\hat{U}_{G E}=\left(\int_{\Theta} U^{-q} \pi^{*}(\Theta \mid t) d \Theta\right)^{-1 / q},
$$

where $q \neq 0$

It is obvious that the integrals in Equations (16)-(18) are complicated. Consequently, the important sampling method and TK method are applied to obtain an approximation for these integrals.

\subsection{Important Sampling Technique}

In this part, we use the important sampling method for estimating the unknown parameters. The posterior distribution presented in (15) can be rewritten as

$$
\omega^{*}\left(\gamma, \alpha, \theta_{1}, b\right) \propto G_{1}(\gamma) \times G_{2}(b) \times G_{3}\left(\theta_{1} \mid \gamma\right) \times G_{4}\left(\alpha \mid \gamma, \theta_{1}, b\right) \times \Delta\left(\gamma, \alpha, \theta_{1}, b\right),
$$

where

$$
\begin{gathered}
G_{1}(\gamma)=\text { GammaDistribution }\left[\mu_{4}-\sum_{i=1}^{k} m_{i}, \lambda_{4}\right], \\
G_{2}(b)=\text { GammaDistribution }\left[\mu_{3},\left(\frac{1}{\lambda_{3}}+\sum_{i=1}^{k} m_{i} \log \left[\psi_{i}\right]\right)^{-1}\right],
\end{gathered}
$$




$$
G_{3}\left(\theta_{1} \mid \gamma\right)=\text { GammaDistribution }\left[\mu_{2}-\frac{1}{\gamma} \sum_{i=1}^{k} m_{i}, \lambda_{2}\right]
$$

and

$$
G_{4}\left(\alpha \mid \gamma, \theta_{1}, b\right)=\text { GammaDistribution }\left[\mu_{1}+\sum_{i=1}^{k} m_{i},\left(\frac{1}{\lambda_{1}}+\sum_{i=1}^{k} \sum_{j=1}^{m_{i}}\left(R_{i j}+1\right) \log \left[1+\left(\frac{t_{i j}}{\psi_{i}^{b}(b+1) \theta_{1}}\right)^{1 / \gamma}\right]\right)^{-1}\right]
$$

Additionally,

$$
\begin{aligned}
\Delta\left(\gamma, \alpha, \theta_{1}, b\right)= & (b+1)^{-\left(\frac{1}{\gamma} \sum_{i=1}^{k} m_{i}\right)} \exp \left\{\sum_{i=1}^{k} m_{i}\left(\frac{1}{\gamma}-1\right)\left(\log \left[t_{i j}\right]-b \log \left[\psi_{i}\right]\right)\right\} \times \\
& \exp \left\{-\sum_{i=1}^{k} \sum_{j=1}^{m_{i}} \log \left[1+\left(\frac{t_{i j}}{\psi_{i}^{b}(b+1) \theta_{1}}\right)^{1 / \gamma}\right]\right\} .
\end{aligned}
$$

Algorithm 1 is used to find the BEs of $\gamma, \alpha, \theta_{1}, b$.

\section{Algorithm 1 Importance Sampling}

1. Generate $\gamma$ from $G_{1}(\gamma)$.

2. Generate $b$ from $G_{2}(b)$.

3. Generate $\theta_{1}$ from $G_{3}\left(\theta_{1} \mid \gamma\right)$.

4. Generate $\alpha$ from $G_{4}\left(\alpha \mid \gamma, \theta_{1}, b\right)$.

5. Repeat steps 1 to $4, N$ times to get $\left(\alpha_{1}, \theta_{11}, \gamma_{1}, b_{1}\right), \ldots,\left(\alpha_{N}, \theta_{1 N}, \gamma_{N}, b_{N}\right)$.

6. The BEs estimate of $u\left(\gamma, \alpha, \theta_{1}, b\right)$ under the SE loss function is

$$
\tilde{u}_{S E}=\frac{\sum_{i=1}^{N} u\left(\gamma, \alpha, \theta_{1}, b\right) \Delta\left(\gamma, \alpha, \theta_{1}, b\right)}{\sum_{i=1}^{N} \Delta\left(\gamma, \alpha, \theta_{1}, b\right)}
$$

7. The BEs estimate of $u\left(\gamma, \alpha, \theta_{1}, b\right)$ under LINEX loss function is

$$
\tilde{u}_{\text {LINEX }}=\frac{-1}{c} \log \left[\frac{\sum_{i=1}^{N} \exp \left\{-c u\left(\gamma, \alpha, \theta_{1}, b\right)\right\} \Delta\left(\gamma, \alpha, \theta_{1}, b\right)}{\sum_{i=1}^{N} \Delta\left(\gamma, \alpha, \theta_{1}, b\right)}\right]
$$

8. The BEs estimate of $u\left(\gamma, \alpha, \theta_{1}, b\right)$ under GE loss function is

$$
\tilde{u}_{G E}=\left[\frac{\sum_{i=1}^{N}\left(u\left(\gamma, \alpha, \theta_{1}, b\right)\right)^{-q} \Delta\left(\gamma, \alpha, \theta_{1}, b\right)}{\sum_{i=1}^{N} \Delta\left(\gamma, \alpha, \theta_{1}, b\right)}\right]^{-1 / q} .
$$

\subsection{Tk-Approximation for Bayesian Estimates}

There are a lot of approximation methods to obtain an approximate value for the integration that depend on the posterior distribution as Monte Carlo methods (Kloek and Van Dijk [31] and Zellner and Rossi [32]) and Lindley method [33], which are computationally intensive. For extra studies where the MCMC and Lindley method are used, see, e.g., [34-37] and [38]. Tierney and Kadane [39] implemented an easily computable integration approximation method that was based on the posterior distribution as the mean and variance of a non-negative parameter or a smooth parameter function that is non-zero within the space of the parameter. The advantage of the Tierney and Kadane (TK) method over Lindley approximation method is that the second method requires the evaluation of the third derivatives of the posterior density or the likelihood function, which can be tiresome and requires great computational precision. 
In this subsection, we use the TK approximation method to compute the Bayesian estimates of the parameters that are based on the SE loss function. TK-method is one of the methods to find the approximate value of the ratio of two integrals as given in Equation (16), which can be rewritten, as follows:

$$
\hat{U}(\Theta)=\frac{\int_{\Theta} U(\Theta) e^{[l(\Theta \mid t)+\rho(\Theta \mid t)]} d \Theta}{\int_{\Theta} e^{[l(\Theta \mid t)+\rho(\Theta \mid t)]} d \Theta},
$$

where $U(\Theta)$ is any function of parameters $\left(\alpha, \gamma, \theta_{1}, b\right), l(\Theta \mid t)$ is defined in (9), and $\rho(\Theta \mid t)$ is the logarithm joint prior distribution that is given by

$$
\begin{aligned}
\rho\left(\alpha, \gamma, \theta_{1}, b \mid t\right) & =\left(\mu_{1}-1\right) \ln (\alpha)+\left(\mu_{2}-1\right) \ln \left(\theta_{1}\right)+\left(\mu_{3}-1\right) \ln (b)+\left(\mu_{4}-1\right) \ln (\gamma) \\
& -\left(\frac{\alpha}{\lambda_{1}}+\frac{\theta_{1}}{\lambda_{2}}+\frac{b}{\lambda_{3}}+\frac{\gamma}{\lambda_{4}}\right) .
\end{aligned}
$$

To obtain an explicit expression for $\hat{U}_{S E}(\Theta)$ using TK approximation, we consider the functions, defined by

$$
\begin{aligned}
\delta(\Theta) & =\frac{l(\Theta \mid t)+\rho(\Theta \mid t)}{n} \\
\delta^{*}(\Theta) & =\delta(\Theta)+\frac{\ln U(\Theta)}{n}
\end{aligned}
$$

Now, assume that the following groups of values $\left(\hat{\alpha}_{\delta}, \hat{\gamma}_{\delta}, \hat{\theta}_{1 \delta}, \hat{b}_{\delta}\right)$ and $\left(\hat{\alpha}_{\delta^{*}}, \hat{\gamma}_{\delta^{*}}, \hat{\theta}_{1 \delta^{*}}, \hat{b}_{\delta^{*}}\right)$ maximize the functions $\delta\left(\alpha, \gamma, \theta_{1}, b\right)$ and $\delta^{*}\left(\alpha, \gamma, \theta_{1}, b\right)$, respectively.

Hence, we approximate $\hat{U}(\Theta)$, as follows:

$$
\hat{U}_{T K}(\Theta)=\sqrt{\frac{\left|\Sigma^{*}\right|}{|\Sigma|}} \exp \left[n\left\{\delta^{*}\left(\hat{\alpha}_{\delta^{*}}, \hat{\gamma}_{\delta^{*}}, \hat{\theta}_{1 \delta^{*}}, \hat{b}_{\delta^{*}}\right)-\delta\left(\hat{\alpha}_{\delta}, \hat{\gamma}_{\delta}, \hat{\theta}_{1 \delta}, \hat{b}_{\delta}\right)\right\}\right]
$$

where $|\Sigma|$ and $\left|\Sigma^{*}\right|$ denote the determinants of negative inverse hessian of $\delta\left(\alpha, \gamma, \theta_{1}, b\right)$ and $\delta^{*}\left(\alpha, \gamma, \theta_{1}, b\right)$, respectively. Moreover, $|\Sigma|$ and $\left|\Sigma^{*}\right|$ are given by

$$
|\Sigma|=\left|\left(\begin{array}{cccc}
\frac{\partial^{2} \delta}{\partial \alpha^{2}} & \frac{\partial^{2} \delta}{\partial \alpha \partial \gamma} & \frac{\partial^{2} \delta}{\partial \alpha \theta_{1}} & \frac{\partial^{2} \delta}{\partial \alpha \partial b} \\
\frac{\partial^{2} \delta}{\partial \gamma \partial \alpha} & \frac{\partial^{2} \delta}{\partial \gamma^{2}} & \frac{\partial^{2} \delta}{\partial \gamma \theta_{1}} & \frac{\partial^{2} \delta}{\partial \gamma \partial b} \\
\frac{\partial^{2} \delta}{\partial \theta_{1} \partial \alpha} & \frac{\partial^{2} \delta}{\partial \theta_{1} \partial \gamma} & \frac{\partial^{2} \delta}{\partial \theta^{2}} & \frac{\partial^{2} \delta}{\partial \theta_{1} \partial b} \\
\frac{\partial^{2} \delta}{\partial b \partial \alpha} & \frac{\partial^{2} \delta}{\partial b \partial \gamma} & \frac{\partial^{2} \delta}{\partial b \partial \theta_{1}} & \frac{\partial^{2} \delta}{\partial b^{2}}
\end{array}\right)\right|^{-1} \quad \& \quad\left|\Sigma^{*}\right|=\left|\left(\begin{array}{cccc}
\frac{\partial^{2} \delta^{*}}{\partial \alpha^{2}} & \frac{\partial^{2} \delta^{*}}{\partial \alpha \partial \gamma} & \frac{\partial^{2} \delta^{*}}{\partial \alpha \partial \theta_{1}} & \frac{\partial^{2} \delta^{*}}{\partial \alpha \partial b} \\
\frac{\partial^{2} \delta^{*}}{\partial \gamma \partial \alpha} & \frac{\partial^{2} \delta^{*}}{\partial \gamma^{2}} & \frac{\partial^{2} \delta^{*}}{\partial \gamma \partial \theta_{1}} & \frac{\partial^{2} \delta^{*}}{\partial \gamma \partial b} \\
\frac{\partial^{2} \delta^{*}}{\partial \theta_{1} \partial \alpha} & \frac{\partial^{2} \delta^{*}}{\partial \theta_{1} \partial \gamma} & \frac{\partial^{2} \delta^{*}}{\partial \theta_{1}^{2}} & \frac{\partial^{2} \delta^{*}}{\partial \theta_{1} \partial b} \\
\frac{\partial^{2} \delta^{*}}{\partial b \partial \alpha} & \frac{\partial^{2} \delta^{*}}{\partial b \partial \gamma} & \frac{\partial^{2} \delta^{*}}{\partial b \partial \theta_{1}} & \frac{\partial^{2} \delta^{*}}{\partial b^{2}}
\end{array}\right)\right|^{-1} \text {. }
$$

\section{Interval Estimation}

In this part of the paper, the approximate and Bootstrap CIs of the parameters $\gamma, \alpha, \theta_{1}$, and $b$ are obtained.

\subsection{Approximate Confidence Intervals}

Based on the asymptotic distribution of the MLEs of the unknown parameters $\gamma, \alpha, \theta_{1}$, and $b$, the normal approximation CIs of the parameters are obtained. The asymptotic distribution of the MLEs of $\left(\gamma, \alpha, \theta_{1}, b\right)$ is given by Miller [40]

$$
\left((\hat{\gamma}-\gamma),\left(\hat{\theta_{1}}-\theta_{1}\right),(\hat{\alpha}-\alpha),(\hat{b}-b)\right) \sim \mathbf{N}(0, \eta),
$$


where $\eta=\left(\kappa_{i j}\right), i, j=1,2,3,4$ is the variance covariance matrix of the unknown parameters. The $100(1-\zeta) \%$ two sided CIs for a general parameter $\vartheta_{i}$ is given by

$$
\left(\hat{\vartheta}_{i L}, \hat{\vartheta}_{i U}\right)=\hat{\vartheta}_{i} \pm Z_{\zeta / 2} \sqrt{\kappa_{i i}}, \quad i=1,2,3,4,
$$

where $\vartheta_{1}=\gamma, \vartheta_{2}=\theta_{1}, \vartheta_{3}=\alpha, \vartheta_{4}=b$, and $Z_{q}$ is the $100 q$-th percentile of a standard normal distribution.

\subsection{Bootstrap Confidence Intervals}

The percentile bootstrap (Boot-p) [41] CIs of the unknown parameters $\gamma, \alpha, \theta_{1}$, and $b$ are obtained. The following steps are used in order to obtain the bootstrap bounds for our distribution parameters. Algorithm 2 is used to find Bootstrap CIs.

\section{Algorithm 2 Bootstrap CIs}

1. Find MLE estimates of the parameters $\alpha, \gamma, \theta_{1}$, and $b$ by solving Equations (10)-(13).

2. Use the estimates in step 1 in order to generate a bootstrap sample $\underline{X}^{*}$ with the same values of the censoring scheme using the algorithm made by [42]. Let us say that the estimates are $\widehat{\alpha}^{*}, \widehat{\gamma}^{*}, \widehat{\theta 1}_{1}^{*}$ and $\widehat{b}^{*}$.

3. Repeat steps 1 and 2, 1000 times.

4. Arrange the estimates ascendingly $\left(\phi_{t}^{(1)}, \phi_{t}^{(2)}, \cdots, \phi_{t}^{(N)}\right), t=1,2,3,4$, where $\phi_{1}=\widehat{\alpha}^{*}, \phi_{2}=$ $\widehat{\gamma}^{*}, \phi_{3}=\widehat{\theta 1}_{1}^{*}, \phi_{4}=\widehat{b}^{*}$. Let $G(x)=P\left(\phi_{t} \leq x\right)$ be the CDF of $\phi_{t}$.

5. Define $\phi_{\text {tboot }}=G^{-1}(x)$ for given $x$. Accordingly, the upper and lower bounds are given by:

$$
\left[\phi_{\text {tboot }}\left(\frac{\beta}{2}\right), \phi_{\text {tboot }}\left(1-\frac{\beta}{2}\right)\right] \text {. }
$$

\section{Application}

In this section, we illustrate the proposed methods by analyzing reliability experiment carried out previously by Zhu [43]. The data in Table 1 were observed from ramp-voltage experiment of miniature light bulbs. In this ramp-voltage experiment, 62 and 61 light bulbs were experimented under ramp-rate $2.01 \mathrm{~V} / \mathrm{h}$, and $2.015 \mathrm{~V} / \mathrm{h}$, respectively, with experimental design stress is $2 \mathrm{~V}$.

To determine whether the real data fit the Pareto-IV distribution, we apply Kolmogorov-Smirnov (K-S) test. Using the MLEs of parameters which are presented in Table 3, the values of K-S statistic and corresponding $p$-values are calculated and shown in Table 2. From results presented in Table 2, we conclude that Pareto-IV distribution is a good fit to the given data according to the $p$-values.

Table 3 includes the MLES and BEs of the model parameters as well as the approximate (App) and Boot CIs for the data set in Table 1. The BEs are obtained while using both TK method and importance sampling method under SE, LINEX, and GE loss functions with $c=-2$ and $q=2$. Furthermore, the values of hyper-parameters used for obtaining BEs are $\mu_{1}=131.8, \mu_{2}=3717.6$, $\mu_{3}=1.3, \mu_{4}=2606.2, \lambda_{1}=0.0027, \lambda_{2}=0.00016, \lambda_{3}=0.00027$, and $\lambda_{4}=0.00061$. 
Table 1. Failure times of miniature light bulbs from ramp-voltage experiment.

\begin{tabular}{cccccccccccc}
\hline \multicolumn{1}{c}{ Stress Volt per Hour 2.01 V/h } & \multicolumn{5}{c}{ Stress Volt per Hour 2.015 V/h } \\
\hline Number & $\mathbf{t}$ & Number & $\mathbf{t}$ & Number & $\mathbf{t}$ & Number & $\mathbf{t}$ & Number & $\mathbf{t}$ & Number & $\mathbf{t}$ \\
\hline 1 & 13.57 & 22 & 72.33 & 43 & 42.06 & 1 & 19.3 & 22 & 49.65 & 43 & 31.00 \\
2 & 19.92 & 23 & 72.60 & 44 & 47.88 & 2 & 23.28 & 23 & 51.42 & 44 & 34.81 \\
3 & 23.3 & 24 & 75.43 & 45 & 54.21 & 3 & 23.50 & 24 & 51.27 & 45 & 36.03 \\
4 & 27.81 & 25 & 75.85 & 46 & 54.55 & 4 & 26.50 & 25 & 53.25 & 46 & 43.08 \\
5 & 31.16 & 26 & 76.20 & 47 & 55.85 & 5 & 27.42 & 26 & 54.25 & 47 & 45.63 \\
6 & 31.56 & 27 & 77.78 & 48 & 56.43 & 6 & 28.32 & 27 & 55.47 & 48 & 46.03 \\
7 & 34.00 & 28 & 79.13 & 49 & 58.86 & 7 & 28.62 & 28 & 56.83 & 49 & 46.33 \\
8 & 46.26 & 29 & 80.65 & 50 & 60.60 & 8 & 30.62 & 29 & 56.17 & 50 & 49.62 \\
9 & 46.41 & 30 & 82.65 & 51 & 62.48 & 9 & 34.42 & 30 & 8.85 & 51 & 49.86 \\
10 & 50.60 & 31 & 90.33 & 52 & 62.81 & 10 & 35.30 & 31 & 11.31 & 52 & 50.66 \\
11 & 56.76 & 32 & 14.51 & 53 & 63.41 & 11 & 35.48 & 32 & 11.83 & 53 & 50.93 \\
12 & 56.85 & 33 & 15.61 & 54 & 63.76 & 12 & 38.30 & 33 & 14.50 & 54 & 51.03 \\
13 & 60.13 & 34 & 15.85 & 55 & 64.18 & 13 & 40.52 & 34 & 14.83 & 55 & 51.73 \\
14 & 65.00 & 35 & 17.73 & 56 & 66.15 & 14 & 43.83 & 35 & 17.73 & 56 & 51.95 \\
15 & 65.86 & 36 & 19.65 & 57 & 66.41 & 15 & 43.00 & 36 & 19.35 & 57 & 52.36 \\
16 & 66.20 & 37 & 21.05 & 58 & 69.91 & 16 & 43.00 & 37 & 25.50 & 58 & 54.78 \\
17 & 66.40 & 38 & 21.20 & 59 & 71.73 & 17 & 43.12 & 38 & 26.15 & 59 & 55.58 \\
18 & 66.80 & 39 & 24.21 & 60 & 72.46 & 18 & 44.43 & 39 & 27.45 & 60 & 55.83 \\
19 & 66.93 & 40 & 24.85 & 61 & 73.78 & 19 & 45.32 & 40 & 27.61 & 61 & 57.13 \\
20 & 68.25 & 41 & 31.18 & 62 & 78.91 & 20 & 47.58 & 41 & 28.05 & & \\
21 & 70.23 & 42 & 35.08 & & & 21 & 47.65 & 42 & 30.96 & & \\
\hline
\end{tabular}

Table 2. K-S statistic and corresponding $p$-values for each stress level.

\begin{tabular}{ccc}
\hline Stress & $\mathbf{2 . 0 1} \mathrm{V} / \mathbf{h}$ & $\mathbf{2 . 0 1 5} \mathrm{V} / \mathbf{h}$ \\
\hline Value of statistic & 0.2258 & 0.125 \\
\hline$p$-value & 0.0846 & 0.7865 \\
\hline
\end{tabular}

Table 3. Maximum likelihood estimates (MLEs), Tierney and Kadane (TK) estimates, importance sampling Bayes estimates (BEs) under square error (SE), linear exponential (LINEX), and general entropy (GE) loss functions, and length of $95 \%$ CIs.

\begin{tabular}{cccccccc}
\hline $\boldsymbol{\Theta}$ & MLE & TK & SE & LINEX & GE & App CI Length & Boot CI length \\
\hline$\hat{\alpha}$ & 6.0973 & 6.09726 & 6.0975 & 6.0975 & 6.09623 & 14.0879 & 22.0187 \\
$\hat{\theta_{1}}$ & 36.5901 & 36.5901 & 36.5818 & 36.5814 & 36.584 & 48.9879 & 41.0014 \\
$\hat{\gamma}$ & 0.3631 & 0.36312 & 0.3598 & 0.3598 & 0.3595 & 0.11123 & 0.1551 \\
$\hat{b}$ & 1.6143 & 1.61439 & 1.6727 & 1.6724 & 1.670 & 3.5197 & 1.63704 \\
\hline
\end{tabular}

\section{Simulation Studies}

The performance of the suggested estimators is studied while using Monte Carlo simulations. The suggested estimators are compared in terms of their relative absolute biases (RABs) and mean square errors (MSEs) for different sample sizes $\left(n_{i}, m_{i}, i=1,2, \ldots, k\right)$, and censoring schemes $\left(R_{i j}, j=\right.$ $\left.1,2, \ldots, m_{i}\right)$. Furthermore, the simulation study is carried out for two designs of the progressive-stress ALT, the first one is the simple ramp-stress with two stress levels $(k=2)$ and their ramp values are $v_{1}=10$ and $v_{2}=16$. The second one is multiple ramp-stress test contains four stress levels $(k=4)$ with ramp values $v_{1}=10, v_{2}=16, v_{3}=20$, and $v_{4}=25$. Tables $4-7$ contain the results that were obtained from the simulation study. Tables 4 and 5 give the values of the MSEs and RABs of MLEs and BEs using the TK method and importance sampling method under SE, LINEX, and GE loss functions with $c=2$ and $q=2$. Tables 6 and 7 introduce the length of CIs and their coverage probabilities. In both Tables 4 and 5, each parameter faces to two horizontal lines, the first containing values of MSEs 
and the second containing values of RABs. The results of simulation studies are obtained using two censoring schemes (CS 1 and CS 2), which are defined as

$$
\text { CS } 1: \quad R_{i j}=\left\{\begin{array}{l}
n_{i}-m_{i}, j=1, \\
0, \text { other wise. }
\end{array}\right.
$$

CS 2: If $m_{i}$ is even, $R_{i j}=\left\{\begin{array}{l}n_{i}-m_{i}, j=\frac{m_{i}}{2}, \\ 0, \text { other wise. }\end{array}\right.$

$$
\text { If } m_{i} \text { is odd, } R_{i j}=\left\{\begin{array}{l}
n_{i}-m_{i}, j=\frac{m_{i}+1}{2}, \\
0, \text { other wise. }
\end{array}\right.
$$

The simulation study is carried out while using Algorithm 3.

\section{Algorithm 3 Steps of the simulation study}

1. Assign the values of $k, \alpha, \theta_{1}, \gamma, b, n_{i}, m_{i}$, and $v_{i}, i=1,2, \ldots, k$.

2. Generate according to the number of levels in the test, random samples of size $m_{i}$ from Uniform $(0,1)$ distribution, $\left(U_{i 1}, U_{i 2}, \ldots, U_{i m_{i}}\right), i=1,2, \ldots, k$.

3. Assign the values of CS, $R_{i j}, i=1,2, \ldots, k$, and $j=1,2, \ldots, m_{i}$ such that $\sum_{j=1}^{m_{i}} R_{i j}=n_{i}-m_{i}$.

4. Set $E_{i j}=U_{i j}^{1 /\left(j+\sum_{d=m_{i}-j+1}^{m_{i}} R_{i d}\right)}, j=1,2, \ldots, m_{i}$, and $i=1,2, \ldots, k$.

5. Obtain $\left(U_{i 1}^{*}, U_{i 2}^{*}, \ldots, U_{i m_{i}}^{*}\right)$, where $U_{i j}^{*}=1-\prod_{d=m_{i}-j+1}^{m_{i}} E_{i d}, j=1,2, \ldots, m_{i}, i=1,2, \ldots, k$.

6. Generate random samples $\left(t_{i 1}, t_{i 2}, \ldots, t_{i m_{i}}\right), i=1,2, \ldots, k$, from (5) as follows:

$$
t_{i j}=\left(\left(\theta_{1}\right)(b+1)\left(\frac{v_{1}}{v_{i}}\right)^{b}\left(-1+\left(1-U_{i j}^{*}\right)^{\frac{-1}{\alpha}}\right)^{\gamma}\right), j=1,2, \ldots, m_{i}, i=1,2, \ldots, k .
$$

7. Use the samples generated in step 6 to obtain the MLEs of the parameters by finding a solution for the non-linear system in ((10)-(13)).

8. Use the samples generated in step 6 to obtain TK Bayes estimates of the parameters from Equation (32).

9. Obtain the BEs using importance sampling method under SE, LINEX, and GE loss functions, according to Algorithm 1.

10. Find the of $95 \%$ normal approximate CIs of the parameters from Equation (33).

11. Find the of $95 \%$ bootstrap CIs of the parameters, using Algorithm 2.

12. Repeat the above steps from step ((2)-(11)), 1000 times.

13. Find the mean values of the RABs, MSEs, and length of CIs for the model parameters. 
Table 4. Mean square errors (MSEs) and relative absolute biases (RABs) for MLEs and BEs of $\alpha, \theta_{1}$, $\gamma$, and $b$ with true values $\left(\gamma=1.4, \alpha=0.5, \theta_{1}=0.7\right.$ and $\left.b=1.2\right)$, values of hyper-parameters $\left(\mu_{1}=196000, \mu_{2}=2500, \mu_{3}=490, \mu_{4}=1440, \lambda_{1}=7.1428 \times 10^{-6}, \lambda_{2}=0.0002, \lambda_{3}=0.0014\right.$, and $\left.\lambda_{4}=0.0008\right), k=2, v_{1}=10$ and $v_{2}=16$.

\begin{tabular}{|c|c|c|c|c|c|c|c|c|}
\hline \multirow{2}{*}{$n_{i}$} & \multirow{2}{*}{$m_{i}$} & \multirow{2}{*}{$C S$} & \multirow{2}{*}{$\theta$} & \multirow{2}{*}{ ML } & \multirow{2}{*}{ TK } & \multicolumn{3}{|c|}{ Important Sampling } \\
\hline & & & & & & SE & LINEX & GE \\
\hline \multirow{16}{*}{$n_{i}= \begin{cases}8 & i=1 \\
9 & i=2\end{cases}$} & \multirow{8}{*}{$m_{i}= \begin{cases}4 & i=1 \\
5 & i=2\end{cases}$} & \multirow{8}{*}{1} & \multirow[b]{2}{*}{$\alpha$} & 0.200304 & $4.37 \times 10^{-7}$ & 0.000112 & 0.000111 & 0.000111 \\
\hline & & & & 0.232240 & 0.001010 & 0.016650 & 0.016650 & 0.016650 \\
\hline & & & \multirow{2}{*}{$\theta_{1}$} & 0.461802 & $4.27 \times 10^{-6}$ & 0.000985 & 0.000985 & 0.000985 \\
\hline & & & & 0.343000 & 0.002450 & 0.036280 & 0.036280 & 0.036280 \\
\hline & & & \multirow{2}{*}{$\beta$} & 0.119454 & $7.40 \times 10^{-8}$ & $9.17 \times 10^{-6}$ & $9.00 \times 10^{-6}$ & $9.00 \times 10^{-6}$ \\
\hline & & & & 0.070850 & 0.000020 & 0.001840 & 0.001840 & 0.001840 \\
\hline & & & \multirow[b]{2}{*}{$b$} & 0.623000 & $6.83 \times 10^{-6}$ & 0.000588 & 0.000588 & 0.000588 \\
\hline & & & & 0.295570 & 0.000210 & 0.000610 & 0.000610 & 0.000610 \\
\hline & \multirow{16}{*}{$m_{i}= \begin{cases}8 & i=1 \\
9 & i=2\end{cases}$} & & & 0.058976 & $1.17 \times 10^{-7}$ & 0.000135 & 0.000135 & 0.000135 \\
\hline & & & $\alpha$ & 0.312900 & 0.000560 & 0.018370 & 0.018370 & 0.018370 \\
\hline & & & & 0.162161 & $3.07 \times 10^{-6}$ & 0.000631 & 0.000631 & 0.000631 \\
\hline & & 2 & $\theta_{1}$ & 0.251000 & 0.001730 & 0.029570 & 0.028600 & 0.029570 \\
\hline & & & $\beta$ & 0.230800 & $4.20 \times 10^{-9}$ & 0.000014 & 0.000014 & 0.000014 \\
\hline & & & $\beta$ & 0.135000 & 0.000010 & 0.002470 & 0.002340 & 0.002340 \\
\hline & & & & 0.210573 & $6.17 \times 10^{-6}$ & 0.000722 & 0.000622 & 0.000733 \\
\hline & & & $b$ & 0.465570 & 0.000590 & 0.017810 & 0.020540 & 0.020440 \\
\hline \multirow{8}{*}{$n_{i}= \begin{cases}8 & i=1 \\
9 & i=2\end{cases}$} & & & & 0.100304 & $3.97 \times 10^{-7}$ & 0.000100 & 0.000001 & 0.000002 \\
\hline & & & a & 0.113224 & 0.001000 & 0.001870 & 0.001880 & 0.001940 \\
\hline & & & & 0.261802 & $3.22 \times 10^{-6}$ & 0.000096 & 0.000116 & 0.000140 \\
\hline & & & $\theta_{1}$ & 0.013320 & 0.002100 & 0.013430 & 0.014810 & 0.016420 \\
\hline & & & & 0.011945 & $5.20 \times 10^{-8}$ & 0.000053 & 0.000890 & 0.000001 \\
\hline & & & $P$ & 0.010850 & 0.000010 & 0.000170 & 0.000176 & 0.000170 \\
\hline & & & & 0.023400 & $5.68 \times 10^{-6}$ & 0.000039 & 0.000029 & 0.000027 \\
\hline & & & $b$ & 0.115570 & 0.000580 & 0.004730 & 0.003950 & 0.003760 \\
\hline & & & & 0.100400 & $8.67 \times 10^{-7}$ & $1.73 \times 10^{-6}$ & $2.00 \times 10^{-6}$ & $2.00 \times 10^{-6}$ \\
\hline & & & $\alpha$ & 0.122400 & 0.001490 & 0.002110 & 0.002410 & 0.002130 \\
\hline $14 i=1$ & $9 i=1$ & & & 0.218020 & $6.99 \times 10^{-6}$ & 0.000332 & 0.000366 & 0.000411 \\
\hline $15 i=2$ & $9 i=2$ & 1 & $\theta_{1}$ & 0.243000 & 0.003030 & 0.025670 & 0.027030 & 0.028660 \\
\hline & & & $\beta$ & 0.114000 & $1.59 \times 10^{-8}$ & $1.06 \times 10^{-6}$ & $2.00 \times 10^{-6}$ & $2.00 \times 10^{-6}$ \\
\hline & & & $P$ & 0.010850 & 0.000020 & 0.000180 & 0.000180 & 0.000180 \\
\hline & & & & 0.323000 & $8.56 \times 10^{-6}$ & 0.000111 & 0.000092 & 0.000088 \\
\hline & & & $b$ & 0.195570 & 0.000650 & 0.008300 & 0.007480 & 0.007280 \\
\hline & & & & 0.189759 & $8.68 \times 10^{-7}$ & $1.73 \times 10^{-6}$ & $2.00 \times 10^{-6}$ & $3.00 \times 10^{-6}$ \\
\hline & & & $\alpha$ & 0.212900 & 0.001490 & 0.002120 & 0.002150 & 0.018370 \\
\hline & & & & 0.142000 & $6.30 \times 10^{-6}$ & 0.000322 & 0.000357 & 0.000401 \\
\hline & & 2 & $\theta_{1}$ & 0.151000 & 0.002870 & 0.025310 & 0.026660 & 0.028290 \\
\hline & & & $B$ & 0.211000 & $1.81 \times 10^{-9}$ & $1.10 \times 10^{-7}$ & $1.14 \times 10^{-7}$ & $1.27 \times 10^{-7}$ \\
\hline & & & $P$ & 0.123000 & 0.000030 & 0.000190 & 0.000180 & 0.000180 \\
\hline & & & $b$ & 0.110573 & $8.22 \times 10^{-6}$ & 0.000124 & 0.000104 & 0.000100 \\
\hline & & & 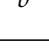 & 0.345700 & 0.000650 & 0.008840 & 0.008020 & 0.007820 \\
\hline & & & & 0.090304 & $1.78 \times 10^{-7}$ & $1.99 \times 10^{-6}$ & $2.00 \times 10^{-6}$ & $2.00 \times 10^{-6}$ \\
\hline & & & $\alpha$ & 0.102400 & 0.002080 & 0.002180 & 0.002190 & 0.002260 \\
\hline $14 i=1$ & $14 i=1$ & & & 0.121802 & $9.63 \times 10^{-6}$ & 0.001540 & 0.000116 & 0.001740 \\
\hline $15 i=2$ & $15 i=2$ & & $\theta_{1}$ & 0.010200 & 0.003440 & 0.056040 & 0.057390 & 0.059110 \\
\hline & & & & 0.011945 & $5.20 \times 10^{-9}$ & $1.30 \times 10^{-7}$ & $19.0 \times 10^{-7}$ & $1.00 \times 10^{-7}$ \\
\hline & & & $\beta$ & 0.010050 & 0.000030 & 0.000110 & 0.000116 & 0.000110 \\
\hline & & & $b$ & 0.013400 & $8.90 \times 10^{-6}$ & 0.000615 & 0.000565 & 0.000554 \\
\hline & & & $b$ & 0.105570 & 0.000638 & 0.002730 & 0.003450 & 0.003260 \\
\hline & & & & 0.070030 & $7.71 \times 10^{-8}$ & 0.000016 & 0.000021 & 0.000117 \\
\hline & & & $\alpha$ & 0.132240 & 0.000120 & 0.001430 & 0.002210 & 0.004970 \\
\hline$\{50 i=1$ & $35 i=1$ & 1 & & 0.036180 & $2.33 \times 10^{-6}$ & 0.000648 & 0.000648 & 0.000648 \\
\hline$\left\{\begin{array}{l}30 i=2 \\
\text { in }\end{array}\right.$ & $10 i=2$ & 1 & $\sigma_{1}$ & 0.093320 & 0.000990 & 0.012800 & 0.012800 & 0.012800 \\
\hline & & & $\beta$ & 0.011945 & $3.67 \times 10^{-9}$ & $6.72 \times 10^{-6}$ & $7.00 \times 10^{-6}$ & $7.00 \times 10^{-6}$ \\
\hline & & & $P$ & 0.070850 & 0.000040 & 0.001600 & 0.001600 & 0.001600 \\
\hline & & & & 0.074542 & $2.16 \times 10^{-6}$ & 0.000588 & 0.000587 & 0.000588 \\
\hline & & & $b$ & 0.195570 & 0.001050 & 0.015910 & 0.015910 & 0.015910 \\
\hline
\end{tabular}


Table 4. Cont.

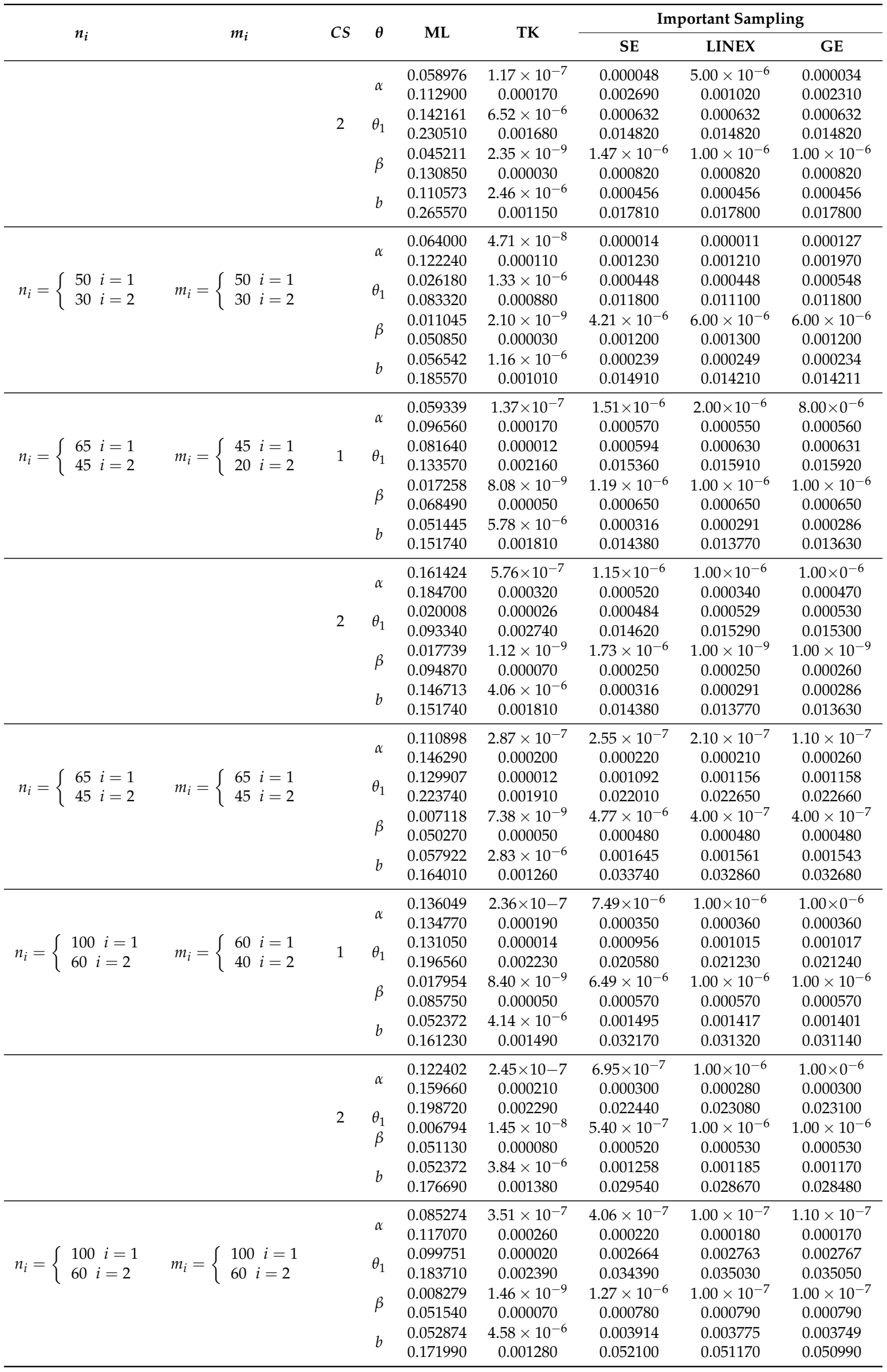


Table 5. Mean square errors (MSEs) and RABs for MLEs and BEs of $\alpha, \theta_{1}, \gamma$, and $b$ with true values $\left(\gamma=1.4, \alpha=0.5, \theta_{1}=0.7\right.$ and $\left.b=1.2\right)$, values of hyper-parameters $\left(\mu_{1}=196000, \mu_{2}=2500, \mu_{3}=490\right.$, $\mu_{4}=1440, \lambda_{1}=7.1428 \times 10^{-6}, \lambda_{2}=0.0002, \lambda_{3}=0.0014$, and $\left.\lambda_{4}=0.0008\right), k=4, v_{1}=10, v_{2}=16$ $v_{3}=20, v_{4}=25$.

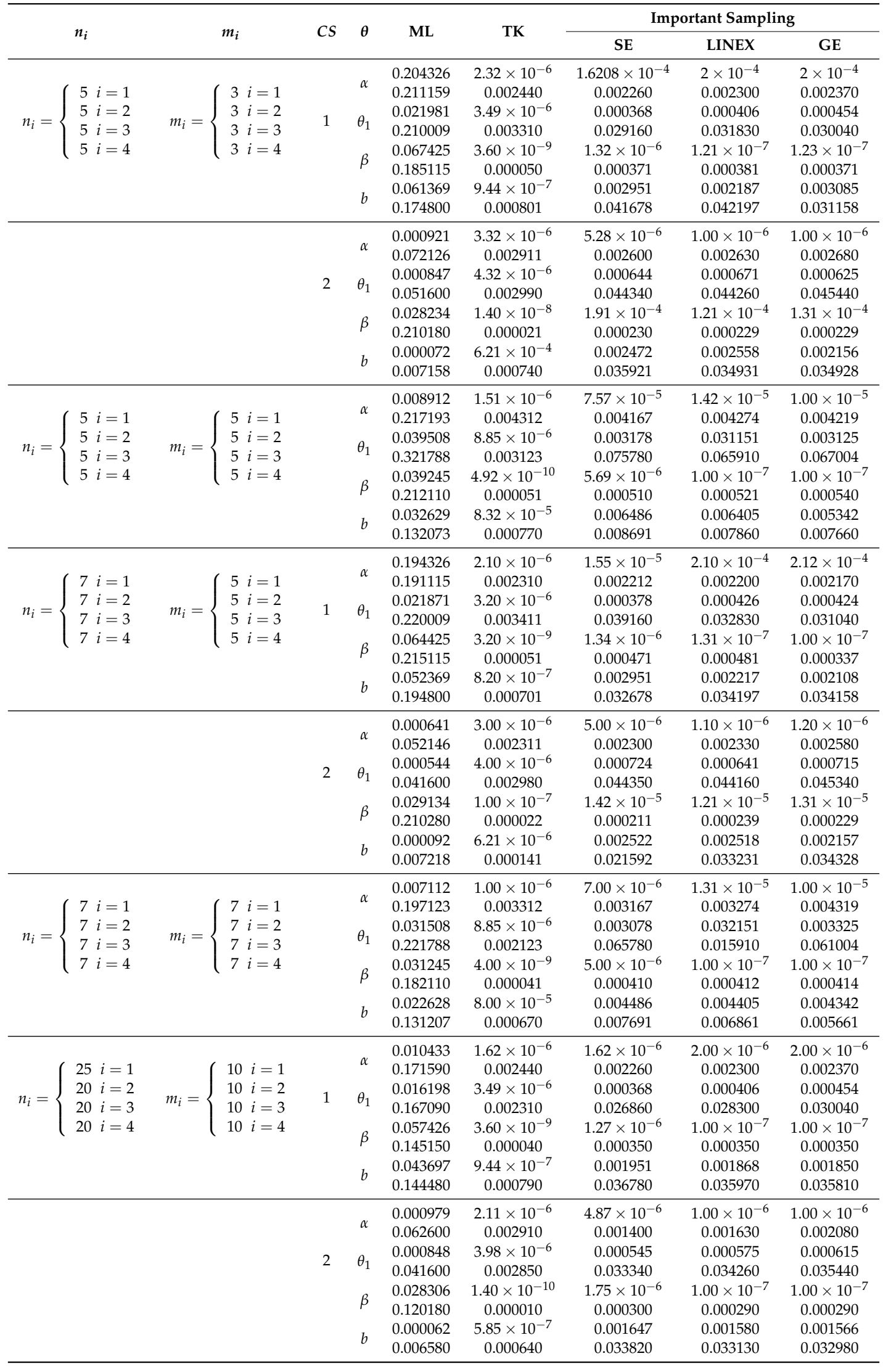


Table 5. Cont.

\begin{tabular}{|c|c|c|c|c|c|c|c|c|}
\hline \multirow{2}{*}{$n_{i}$} & \multirow{2}{*}{$m_{i}$} & \multirow{2}{*}{ CS } & \multirow{2}{*}{$\theta$} & \multirow{2}{*}{ ML } & \multirow{2}{*}{ TK } & \multicolumn{3}{|c|}{ Important Sampling } \\
\hline & & & & & & SE & LINEX & GE \\
\hline$n_{i}= \begin{cases}25 & i=1 \\
20 & i=2 \\
20 & i=3 \\
20 & i=4\end{cases}$ & $m_{i}= \begin{cases}25 & i=1 \\
20 & i=2 \\
20 & i=3 \\
20 & i=4\end{cases}$ & & $\theta_{1}$ & $\begin{array}{l}0.008938 \\
0.171930 \\
0.029508 \\
0.217880 \\
0.039245 \\
0.135110 \\
0.017628 \\
0.107340\end{array}$ & $\begin{array}{c}1.52 \times 10^{-6} \\
0.002200 \\
8.85 \times 10^{-6} \\
0.003830 \\
4.92 \times 10^{-10} \\
0.000040 \\
8.96 \times 10^{-7} \\
0.000780\end{array}$ & $\begin{array}{c}7.57 \times 10^{-7} \\
0.001670 \\
0.002078 \\
0.064780 \\
5.68 \times 10^{-6} \\
0.000400 \\
0.005188 \\
0.059090\end{array}$ & $\begin{array}{c}1.00 \times 10^{-6} \\
0.001740 \\
0.002151 \\
0.065910 \\
1.00 \times 10^{-7} \\
0.000400 \\
0.005049 \\
0.058290\end{array}$ & $\begin{array}{c}1.00 \times 10^{-6} \\
0.001880 \\
0.002249 \\
0.067400 \\
1.00 \times 10^{-7} \\
0.000400 \\
0.005024 \\
0.058140\end{array}$ \\
\hline $\begin{array}{l}40 i=1 \\
30 i=2 \\
30 i=3 \\
30 i=4\end{array}$ & $\begin{array}{l}25 i=1 \\
20 \quad i=2 \\
20 \quad i=3 \\
20 \quad i=4\end{array}$ & 1 & $\theta_{1}$ & $\begin{array}{l}0.003908 \\
0.120590 \\
0.022139 \\
0.202780 \\
0.028294 \\
0.089840 \\
0.056264 \\
0.170840\end{array}$ & $\begin{array}{c}2.01 \times 10^{-6} \\
0.002550 \\
0.000016 \\
0.004530 \\
1.16 \times 10^{-8} \\
0.000060 \\
8.13 \times 10^{-7} \\
0.000730\end{array}$ & $\begin{array}{c}0.000011 \\
0.005610 \\
0.001389 \\
0.046120 \\
3.19 \times 10^{-6} \\
0.001010 \\
0.005337 \\
0.059590\end{array}$ & $\begin{array}{c}0.000011 \\
0.005530 \\
0.001456 \\
0.047610 \\
3.00 \times 10^{-7} \\
0.001000 \\
0.005218 \\
0.058890\end{array}$ & $\begin{array}{c}0.000011 \\
0.005460 \\
0.001548 \\
0.049550 \\
3.00 \times 10^{-7} \\
0.001000 \\
0.005197 \\
0.058760\end{array}$ \\
\hline & & 2 & $\theta_{1}$ & $\begin{array}{l}0.002635 \\
0.080740 \\
0.016658 \\
0.130320 \\
0.031085 \\
0.119090 \\
0.028543 \\
0.130920\end{array}$ & $\begin{array}{c}3.33 \times 10^{-6} \\
0.003140 \\
9.89 \times 10^{-6} \\
0.003810 \\
4.62 \times 10^{-9} \\
0.000050 \\
9.39 \times 10^{-7} \\
0.000730\end{array}$ & $\begin{array}{c}.01 \times 10^{-6} \\
0.003050 \\
0.002737 \\
0.074270 \\
6.60 \times 10^{-7} \\
0.000490 \\
0.004388 \\
0.055060\end{array}$ & $\begin{array}{c}4.01 \times 10^{-6} \\
0.003160 \\
0.002806 \\
0.075210 \\
1.00 \times 10^{-7} \\
0.000490 \\
0.004264 \\
0.054280\end{array}$ & $\begin{array}{c}5.00 \times 10^{-6} \\
0.003390 \\
0.002901 \\
0.076520 \\
1.00 \times 10^{-7} \\
0.000490 \\
0.004242 \\
0.054130\end{array}$ \\
\hline $\begin{array}{l}40 i=1 \\
30 i=2 \\
30 i=3 \\
30 i=4\end{array}$ & $\begin{array}{l}40 i=1 \\
30 i=2 \\
30 i=3 \\
30 i=4\end{array}$ & & $\theta_{1}$ & $\begin{array}{l}0.003945 \\
0.125620 \\
0.051662 \\
0.324700 \\
0.002229 \\
0.033720 \\
0.045313 \\
0.177390\end{array}$ & $\begin{array}{c}1.82 \times 10^{-6} \\
0.002700 \\
0.000043 \\
0.009350 \\
1.01 \times 10^{-9} \\
0.000001 \\
5.02 \times 10^{-7} \\
0.000590\end{array}$ & $\begin{array}{c}9.92 \times 10^{-7} \\
0.001990 \\
0.008010 \\
0.127860 \\
1.05 \times 10^{-7} \\
0.000730 \\
0.010600 \\
0.085800\end{array}$ & $\begin{array}{c}1.00 \times 10^{-6} \\
0.002060 \\
0.008105 \\
0.128610 \\
1.00 \times 10^{-7} \\
0.000740 \\
0.010347 \\
0.084770\end{array}$ & $\begin{array}{c}1.00 \times 10^{-6} \\
0.002200 \\
0.008248 \\
0.129740 \\
1.00 \times 10^{-7} \\
0.000740 \\
0.010308 \\
0.084610\end{array}$ \\
\hline $\begin{array}{l}80 i=1 \\
60 i=2 \\
60 i=3 \\
60 i=4\end{array}$ & $\begin{array}{l}60 i=1 \\
45 i=2 \\
45 i=3 \\
45 i=4\end{array}$ & 1 & $\theta_{1}$ & $\begin{array}{l}0.005745 \\
0.124970 \\
0.009045 \\
0.122810 \\
0.045596 \\
0.122530 \\
0.047806 \\
0.117930\end{array}$ & $\begin{array}{c}5.87 \times 10^{-6} \\
0.003280 \\
0.000024 \\
0.005420 \\
6.58 \times 10^{-9} \\
0.000050 \\
2.16 \times 10^{-6} \\
0.001140\end{array}$ & $\begin{array}{c}8.29 \times 10^{-6} \\
0.004920 \\
0.017979 \\
0.190050 \\
5.43 \times 10^{-6} \\
0.001340 \\
0.019736 \\
0.114960\end{array}$ & $\begin{array}{c}8.00 \times 10^{-6} \\
0.004860 \\
0.018045 \\
0.190400 \\
5.00 \times 10^{-6} \\
0.001340 \\
0.019666 \\
0.114740\end{array}$ & $\begin{array}{c}8.00 \times 10^{-6} \\
0.004740 \\
0.018163 \\
0.191030 \\
5.00 \times 10^{-6} \\
0.001340 \\
0.019657 \\
0.114710\end{array}$ \\
\hline & & 2 & $\theta_{1}$ & $\begin{array}{l}0.002655 \\
0.093450 \\
0.018653 \\
0.170830 \\
0.006572 \\
0.047190 \\
0.041606 \\
0.151820\end{array}$ & $\begin{array}{c}8.71 \times 10^{-6} \\
0.004660 \\
0.000039 \\
0.006340 \\
7.98 \times 10^{-9} \\
0.000060 \\
2.21 \times 10^{-6} \\
0.001130\end{array}$ & $\begin{array}{c}0.000023 \\
0.007180 \\
0.022114 \\
0.211600 \\
1.88 \times 10^{-6} \\
0.000690 \\
0.016220 \\
0.105700\end{array}$ & $\begin{array}{c}0.000024 \\
0.007280 \\
0.022194 \\
0.211990 \\
2.00 \times 10^{-6} \\
0.000680 \\
0.016126 \\
0.105400\end{array}$ & $\begin{array}{c}0.000025 \\
0.007470 \\
0.022340 \\
0.212710 \\
2.00 \times 10^{-6} \\
0.000680 \\
0.016114 \\
0.105360\end{array}$ \\
\hline $\begin{array}{l}80 i=1 \\
60 i=2 \\
60 i=3 \\
60 i=4\end{array}$ & $\begin{array}{l}80 i=1 \\
60 i=2 \\
60 i=3 \\
60 i=4\end{array}$ & & $\theta_{1}$ & $\begin{array}{l}0.002446 \\
0.092370 \\
0.020805 \\
0.157070 \\
0.028698 \\
0.098050 \\
0.054623 \\
0.073830\end{array}$ & $\begin{array}{c}2.01 \times 10^{-6} \\
0.002430 \\
5.39 \times 10^{-6} \\
0.003020 \\
9.49 \times 10^{-9} \\
0.000050 \\
1.94 \times 10^{-6} \\
0.001140\end{array}$ & $\begin{array}{c}0.000062 \\
0.012940 \\
0.019193 \\
0.194610 \\
2.28 \times 10^{-6} \\
0.000900 \\
0.021122 \\
0.166880\end{array}$ & $\begin{array}{c}0.000062 \\
0.012900 \\
0.019251 \\
0.194930 \\
2.00 \times 10^{-6} \\
0.000910 \\
0.020898 \\
0.166260\end{array}$ & $\begin{array}{c}0.000062 \\
0.012840 \\
0.019352 \\
0.195470 \\
2.00 \times 10^{-6} \\
0.000910 \\
0.020873 \\
0.166220\end{array}$ \\
\hline
\end{tabular}


Table 6. Lengths and coverage probabilities of $95 \%$ approximate and bootstrap CIs of $\alpha, \theta_{1}, \gamma$ and $b$ with true values $\left(\gamma=1.4, \alpha=0.5, \theta_{1}=0.7\right.$ and $\left.b=1.2\right), k=2, v_{1}=10$, and $v_{2}=16$.

\begin{tabular}{|c|c|c|c|c|c|c|c|c|}
\hline \multirow{2}{*}{\multicolumn{2}{|c|}{$n_{i}$}} & \multirow{2}{*}{$m_{i}$} & \multirow{2}{*}{$C S$} & \multirow{2}{*}{$\theta$} & \multicolumn{2}{|r|}{ Approximate CI } & \multicolumn{2}{|r|}{ Bootstrap CI } \\
\hline & & & & & Length & Coverage Probability & Length & Coverage Probability \\
\hline \multirow{8}{*}{$n_{i}=\{$} & \multirow{8}{*}{$\begin{array}{ll}8 & i=1 \\
9 & i=2\end{array}$} & \multirow{8}{*}{$m_{i}= \begin{cases}4 & i=1 \\
5 & i=2\end{cases}$} & \multirow{4}{*}{1} & $\alpha$ & 3.895 & 0.827 & 1.12 & 0.700 \\
\hline & & & & $\theta_{1}$ & 1.436 & 0.780 & 0.903 & 0.875 \\
\hline & & & & $\beta$ & 1.469 & 0.870 & 0.679 & 0.756 \\
\hline & & & & $b$ & 2.480 & 0.780 & 0.869 & 0.788 \\
\hline & & & \multirow{4}{*}{2} & $\alpha$ & 3.528 & 0.820 & 0.982 & 0.661 \\
\hline & & & & $\theta_{1}$ & 1.427 & 0.840 & 0.998 & 0.712 \\
\hline & & & & $\beta$ & 1.403 & 0.891 & 0.678 & 0.870 \\
\hline & & & & $b$ & 2.600 & 0.890 & 0.760 & 0.670 \\
\hline \multirow{4}{*}{\multicolumn{2}{|c|}{$n_{i}= \begin{cases}8 & i=1 \\
9 & i=2\end{cases}$}} & \multirow{4}{*}{$m_{i}= \begin{cases}8 & i=1 \\
9 & i=2\end{cases}$} & & $\alpha$ & 3.822 & 0.891 & 1.111 & 0.890 \\
\hline & & & & $\theta_{1}$ & 1.120 & 0.880 & 0.991 & 0.955 \\
\hline & & & & $\beta$ & 1.269 & 0.890 & 0.659 & 0.796 \\
\hline & & & & $b$ & 2.100 & 0.878 & 0.819 & 0.912 \\
\hline \multirow{8}{*}{\multicolumn{2}{|c|}{$n_{i}= \begin{cases}14 & i=1 \\
15 & i=2\end{cases}$}} & & & $\alpha$ & 2.891 & 0.910 & 1.101 & 0.841 \\
\hline & & $9 i=1$ & & $\theta_{1}$ & 1.236 & 0.880 & 0.802 & 0.885 \\
\hline & & $9 i=2$ & 1 & $\beta$ & 1.1698 & 0.890 & 0.590 & 0.816 \\
\hline & & & & $b$ & 2.280 & 0.940 & 0.789 & 0.818 \\
\hline & & & & $\alpha$ & 2.800 & 0.860 & 0.821 & 0.861 \\
\hline & & & 2 & $\theta_{1}$ & 1.427 & 0.840 & 0.998 & 0.712 \\
\hline & & & 2 & $\beta$ & 1.410 & 0.811 & 0.547 & 0.810 \\
\hline & & & & $b$ & 2.100 & 0.880 & 0.560 & 0.801 \\
\hline & & & & $\alpha$ & 2.910 & 0.901 & 0.990 & 0.910 \\
\hline & $14 i=1$ & $14 i=1$ & & $\theta_{1}$ & 0.960 & 0.890 & 0.810 & 0.960 \\
\hline$n_{i}=$ & $15 i=2$ & $15 i=2$ & & $\beta$ & 1.026 & 0.930 & 0.830 & 0.830 \\
\hline & & & & $b$ & 1.980 & 0.780 & 0.719 & 0.921 \\
\hline & & & & $\alpha$ & 2.940 & 0.929 & 0.942 & 1 \\
\hline & $50 i=1$ & $20 i=1$ & & $\theta_{1}$ & 1.378 & 0.880 & 0.883 & 0.975 \\
\hline & $35 i=2$ & $m_{i}= \begin{cases}10 & i=2\end{cases}$ & 1 & $\beta$ & 1.369 & 0.950 & 0.579 & 0.990 \\
\hline & & & & $b$ & 1.969 & 0.900 & 0.690 & 0.980 \\
\hline & & & & $\alpha$ & 2.245 & 0.920 & 0.892 & 1 \\
\hline & & & & $\theta_{1}$ & 1.2175 & 0.900 & 0.856 & 0.900 \\
\hline & & & 2 & $\beta$ & 1.209 & 0.950 & 0.489 & 0.990 \\
\hline & & & & $b$ & 2.208 & 1 & 0.68 & 0.98 \\
\hline & & & & $\alpha$ & 2.045 & 0.990 & 0.792 & 1 \\
\hline & $50 i=1$ & $50 i=1$ & & $\theta_{1}$ & 1.110 & 0.990 & 0.756 & 0.980 \\
\hline & $35 i=2$ & $m_{i}=\left\{\begin{array}{l}35 i=2 \\
\end{array}\right.$ & & $\beta$ & 1.009 & 0.890 & 0.970 & 0.990 \\
\hline & & & & $b$ & 2.008 & 0.880 & 0.610 & 0.990 \\
\hline & & & & $\alpha$ & 1.627 & 1 & 0.952 & 1 \\
\hline & $65 i=1$ & $45 i=1$ & & $\theta_{1}$ & 1.126 & 1 & 0.842 & 1 \\
\hline & $45 i=2$ & $20 i=2$ & 1 & $\beta$ & 0.955 & 1 & 0.470 & 1 \\
\hline & & & & $b$ & 2.175 & 1 & 0.676 & 1 \\
\hline & & & & $\alpha$ & 2.625 & 1 & 1.043 & 1 \\
\hline & & & & $\theta_{1}$ & 1.420 & 1 & 0.796 & 1 \\
\hline & & & 2 & $\beta$ & 1.597 & 1 & 0.484 & 1 \\
\hline & & & & $b$ & 1.731 & 1 & 0.651 & 1 \\
\hline & & & & $\alpha$ & 0.772 & 0.900 & 0.913 & 1 \\
\hline & $65 i=1$ & $65 i=1$ & & $\theta_{1}$ & 0.681 & 1 & 0.839 & 1 \\
\hline & $45 i=2$ & $45 i=2$ & & $\beta$ & 0.766 & 1 & 0.462 & 1 \\
\hline & & & & $b$ & 1.829 & 1 & 0.674 & 1 \\
\hline & & & & $\alpha$ & 0.874 & 0.900 & 1.030 & 1 \\
\hline$n_{i}=$ & $100 i=1$ & $60 i=1$ & & $\theta_{1}$ & 0.663 & 1 & 0.865 & 1 \\
\hline$n_{i}=\{$ & $60 i=2$ & $m_{i}=\left\{\begin{array}{l}40 i=2 \\
\end{array}\right.$ & 1 & $\beta$ & 0.721 & 1 & 0.485 & 1 \\
\hline & & & & $b$ & 1.825 & 1 & 0.67491 & 1 \\
\hline & & & & $\alpha$ & 2.003 & 1 & 0.926 & 1 \\
\hline & & & 2 & $\theta_{1}$ & 1.153 & 1 & 0.846 & 1 \\
\hline & & & 2 & $\beta$ & 0.895 & 1 & 0.438 & 1 \\
\hline & & & & $b$ & 2.122 & 1 & 0.690 & 1 \\
\hline & & & & $\alpha$ & 0.635 & 0.900 & 0.950 & 1 \\
\hline$n_{i}=\{$ & $60 i=2$ & $m_{i}=\left\{\begin{array}{l}100 i=1 \\
60 i=2\end{array}\right.$ & & $\theta_{1}$ & 0.572 & 0.900 & 0.842 & 1 \\
\hline & $60 \imath=2$ & $(60 i=2$ & & $\beta$ & 0.636 & 1 & 0.3878 & 1 \\
\hline & & & & $b$ & 1.402 & 1 & 0.684 & 1 \\
\hline
\end{tabular}


Table 7. Lengths and coverage probabilities of $95 \%$ approximate and bootstrap CIs of $\alpha, \theta_{1}, \gamma$ and $b$ with true values $\left(\gamma=1.4, \alpha=0.5, \theta_{1}=0.7\right.$ and $\left.b=1.2\right), k=4, v_{1}=10, v_{2}=16, v_{3}=20$ and $v_{4}=25$.

\begin{tabular}{|c|c|c|c|c|c|c|c|c|c|}
\hline & \multirow{2}{*}{$n_{i}$} & \multirow{2}{*}{\multicolumn{2}{|c|}{$m_{i}$}} & \multirow{2}{*}{$C S$} & \multirow{2}{*}{$\theta$} & \multicolumn{2}{|r|}{ Approximate CI } & \multicolumn{2}{|r|}{ Bootstrap CI } \\
\hline & & & & & & Length & Coverage Probability & Length & Coverage Probability \\
\hline \multirow{8}{*}{$n_{i}=$} & $5 i=1$ & & $3 i=1$ & \multirow{4}{*}{1} & $\alpha$ & 0.632 & 0.900 & 0.2681 & 0.920 \\
\hline & $5 i=2$ & & $3 i=2$ & & $\theta_{1}$ & 2.849 & 0.911 & 0.416 & 0.913 \\
\hline & $5 i=3$ & $m_{i}=$ & $3 i=3$ & & $\beta$ & 1.261 & 0.921 & 0.801 & 0.711 \\
\hline & $5 i=4$ & & $3 i=4$ & & $b$ & 4.891 & 0.7140 & 0.713 & 0.816 \\
\hline & & & & \multirow{4}{*}{2} & $\alpha$ & 0.718 & 0.808 & 0.292 & 0.841 \\
\hline & & & & & $\theta_{1}$ & 1.927 & 0.910 & 0.312 & 0.970 \\
\hline & & & & & $\beta$ & 1.328 & 0.900 & 0.617 & 0.910 \\
\hline & & & & & $b$ & 3.922 & 0.820 & 0.718 & 0.990 \\
\hline \multirow{4}{*}{$n_{i}=\{$} & $5 i=1$ & \multirow{4}{*}{$m_{i}=$} & $5 i=1$ & & $\alpha$ & 0.612 & 0.951 & 0.240 & 0.912 \\
\hline & $5 i=2$ & & $5 i=2$ & & $\theta_{1}$ & 2.517 & 0.720 & 0.389 & 0.912 \\
\hline & $5 i=3$ & & $5 i=3$ & & $\beta$ & 0.921 & 1 & 0.611 & 1 \\
\hline & $5 i=4$ & & $5 i=4$ & & $b$ & 3.219 & 1 & 0.718 & 1 \\
\hline \multirow{8}{*}{$n_{i}=\{$} & $7 i=1$ & & $(5 i=1$ & \multirow{4}{*}{1} & $\alpha$ & 0.532 & 0.910 & 0.241 & 0.970 \\
\hline & $7 i=2$ & & $5 i=2$ & & $\theta_{1}$ & 2.749 & 0.920 & 0.316 & 0.930 \\
\hline & $7 i=3$ & $m_{i}=$ & $5 i=3$ & & $\beta$ & 1.211 & 0.981 & 0.6201 & 0.911 \\
\hline & $7 i=4$ & & $5 i=4$ & & $b$ & 4.291 & 0.8140 & 0.813 & 0.816 \\
\hline & & & & & $\alpha$ & 0.718 & 0.808 & 0.292 & 0.841 \\
\hline & & & & & $\theta_{1}$ & 1.927 & 0.910 & 0.312 & 0.970 \\
\hline & & & & 2 & $\beta$ & 1.328 & 0.900 & 0.617 & 0.910 \\
\hline & & & & & $b$ & 3.922 & 0.820 & 0.718 & 0.990 \\
\hline & $7 i=1$ & & $7 i=1$ & & $\alpha$ & 0.412 & 0.961 & 0.210 & 0.922 \\
\hline & $7 i=2$ & & $7 i=2$ & & $\theta_{1}$ & 2.417 & 0.820 & 0.319 & 0.912 \\
\hline$n_{i}$ & $7 i=3$ & $m_{i}$ & $7 i=3$ & & $\beta$ & 0.911 & 1 & 0.611 & 1 \\
\hline & $7 i=4$ & & $7 i=4$ & & $b$ & 3.119 & 1 & 0.618 & 1 \\
\hline & $25 i=1$ & & $10 i=1$ & & $\alpha$ & 0.722 & 0.950 & 0.242 & 0.980 \\
\hline & $20 i=2$ & & $10 i=2$ & & $\theta_{1}$ & 2.489 & 1 & 0.391 & 1 \\
\hline & $20 i=3$ & $m_{i}=$ & $10 i=3$ & 1 & $\beta$ & 1.091 & 1 & 0.708 & 1 \\
\hline & $20 i=4$ & & $10 i=4$ & & $b$ & 4.854 & 0.940 & 0.683 & 0.990 \\
\hline & & & & & $\alpha$ & 0.742 & 0.88 & 0.272 & 0.99 \\
\hline & & & & 2 & $\theta_{1}$ & 1.726 & 0.940 & 0.362 & 0.970 \\
\hline & & & & 2 & $\beta$ & 1.188 & 0.900 & 0.567 & 0.940 \\
\hline & & & & & $b$ & 3.872 & 0.920 & 0.658 & 0.990 \\
\hline & $25 i=1$ & & $25 i=1$ & & $\alpha$ & 0.777 & 1 & 0.280 & 1 \\
\hline & $20 i=2$ & & $20 i=2$ & & $\theta_{1}$ & 2.216 & 0.610 & 0.381 & 1 \\
\hline$n_{i}=$ & $20 i=3$ & $m_{i}=$ & $20 i=3$ & & $\beta$ & 0.991 & 1 & 0.581 & 1 \\
\hline & $20 i=4$ & & $20 i=4$ & & $b$ & 3.309 & 1 & 0.658 & 1 \\
\hline & $40 i=1$ & & $25 i=1$ & & $\alpha$ & 0.778 & 0.860 & 0.280 & 0.960 \\
\hline & $30 i=2$ & & $20 i=2$ & & $\theta_{1}$ & 2.232 & 0.820 & 0.397 & 0.983 \\
\hline & $30 i=3$ & $m_{i}=$ & $20 i=3$ & 1 & $\beta$ & 0.893 & 0.880 & 0.586 & 0.990 \\
\hline & $30 i=4$ & & $20 i=4$ & & $b$ & 3.286 & 0.670 & 0.674 & 0.987 \\
\hline & & & & & $\alpha$ & 0.764 & 0.880 & 0.238 & 0.970 \\
\hline & & & & 2 & $\theta_{1}$ & 2.122 & 0.920 & 0.392 & 0.900 \\
\hline & & & & 2 & $\beta$ & 0.853 & 0.838 & 0.581 & 0.929 \\
\hline & & & & & $b$ & 3.218 & 0.880 & 0.674 & 0.987 \\
\hline & $40 i=1$ & & $40 i=1$ & & $\alpha$ & 0.778 & 1 & 0.280 & 1 \\
\hline & $30 i=2$ & & $30 i=2$ & & $\theta_{1}$ & 2.216 & 1 & 0.381 & 1 \\
\hline$n_{i}=$ & $30 i=3$ & $m_{i}=$ & $30 i=3$ & & $\beta$ & 0.919 & 1 & 0.581 & 1 \\
\hline & $30 i=4$ & & $30 i=4$ & & $b$ & 2.900 & 1 & 0.658 & 1 \\
\hline & $80 i=1$ & & $60 i=1$ & & $\alpha$ & 0.410 & 0.910 & 0.238 & 0.975 \\
\hline & $60 i=2$ & & $45 i=2$ & 1 & $\theta_{1}$ & 1.181 & 1 & 0.394 & 1 \\
\hline & $60 i=3$ & $m_{i}=$ & $45 i=3$ & & $\beta$ & 0.648 & 1 & 0.441 & 1 \\
\hline & $60 i=4$ & & $45 i=4$ & & $b$ & 2.437 & 1 & 0.666 & 1 \\
\hline & & & & & $\alpha$ & 0.393 & 1 & 0.200 & 1 \\
\hline & & & & 2 & $\theta_{1}$ & 1.146 & 1 & 0.398 & 1 \\
\hline & & & & & $\beta$ & 0.588 & 0.800 & 0.444 & 1 \\
\hline & & & & & $b$ & 2.052 & 1 & 0.690 & 1 \\
\hline & $80 i=1$ & & $80 i=1$ & & $\alpha$ & 0.382 & 1 & 0.200 & 1 \\
\hline & $60 i=2$ & & $60 i=2$ & & $\theta_{1}$ & 1.210 & 1 & 0.364 & 1 \\
\hline & $60 i=3$ & & $60 i=3$ & & $\beta$ & 0.615 & 1 & 0.416 & 1 \\
\hline & $60 i=4$ & & $60 i=4$ & & $b$ & 2.054 & 1 & 0.655 & 1 \\
\hline
\end{tabular}


From the results in Tables 4-7, we concluded that

1. The BEs of the model parameters give more accurate results than MLEs according to MSEs and RABs.

2. The TK method gives more accurate results than the importance sampling method in most cases.

3. With respect to the importance sampling method, in most of the iteration LINEX loss function had the best values when compared to SE and GE loss functions.

4. The length of bootstrap CIs is smaller than the corresponding approximate CIs.

5. The coverage probability of bootstrap CIs of the four parameters is greater than the corresponding probability of approximate CIs.

\section{Conclusions}

This study addressed statistical inference that was based on progressively type-II censored data from Pareto-IV distribution under progressive-stress accelerated life test. The statistical inference that was covered in this paper includes: estimating the unknown parameters of Pareto-IV distribution by several methods, including the classical method and Bayesian methods. In this context, two methods were used to find Bayes estimates, namely the TK approximation method and importance sampling method. A comprehensive simulation study was conducted to compare different estimation methods; from them, we concluded that Bayes' estimation methods are more accurate than the classical method. Furthermore, TK method is more accurate than importance sampling method according to MSEs and RABs. Regarding to the interval estimation, the normal approximate and bootstrap confidence intervals were obtained. We have deduced from the simulation study that the length of bootstrap CIs is smaller than approximate CIs. Moreover, the coverage probability of bootstrap CIs is greater than the corresponding approximate CIs. We did not rely only on simulation studies, but also analyzed real data from ramp-voltage experiment of miniature light bulbs in order to clarify the different estimation methods.

Author Contributions: All authors contributed equally to this work. All authors have read and agreed to the published version of the manuscript.

Funding: This research received no external funding.

Acknowledgments: The authors are sincerely grateful to the referees and to the Editor-in-Chief for their many constructive comments and careful reading of the paper.

Conflicts of Interest: The authors declare no conflict of interest.

\section{Abbreviations}

The following abbreviations are used in this manuscript:

$\begin{array}{ll}\text { ALT } & \text { Accelerated life test } \\ \text { MLE } & \text { Maximum likelihood estimation } \\ \text { BE } & \text { Bayesian estimation } \\ \text { TK } & \text { Tierney and Kadane } \\ \text { CI } & \text { Confidence interval } \\ \text { Pareto-IV } & \text { Pareto distribution of type IV } \\ \text { CS } & \text { Censoring scheme } \\ \text { CDF } & \text { Cumulative distribution function } \\ \text { PDF } & \text { Probability density function } \\ \text { SE } & \text { Squared error } \\ \text { LINEX } & \text { Linear exponential } \\ \text { GE } & \text { Generalized entropy } \\ \text { K-S } & \text { Kolmogorov-Smirnov } \\ \text { MSE } & \text { Mean square error } \\ \text { RAB } & \text { Relative absolute bias }\end{array}$




\section{References}

1. Nelson, W. Accelerated Testing: Statistical Models, Test Plans and Data Analysis; Wiley: New York, NY, USA, 1990.

2. Guan, Q.; Tang, Y.; Fu, J.; Xu, A. Optimal multiple constant-stress accelerated life tests for generalized exponential distribution. Commun. Stat. Simul. Comput. 2014, 43, 1852-1865. [CrossRef]

3. Jaheen, Z.F.; Moustafa, H.M.; Abd El-Monem, G.H. Bayes inference in constant partially accelerated life tests for the generalized exponential distribution with progressive censoring. Commun. Stat. Theory Methods 2014, 43, 2973-2988. [CrossRef]

4. Kim, C.M.; Bai, D.S. Analysis of accelerated life test data under two failure modes. Int. J. Reliab. Qual. Saf. Eng. 2002, 9, 111-125. [CrossRef]

5. Mohie El-Din, M.M.; Abu-Youssef, S.E.; Ali, N.S.; Abd El-Raheem, A.M. Estimation in constant-stress accelerated life tests for extension of the exponential distribution under progressive censoring. Metron 2016, 74, 253-273. [CrossRef]

6. Mohie El-Din, M.M.; Abu-Youssef, S.E.; Ali, N.S.A.; Abd El-Raheem, A.M. Optimal plans of constant-stress accelerated life tests for the Lindley distribution. J. Test. Eval. 2017, 45, 1463-1475. [CrossRef]

7. Watkins, A.J.; John, A.M. On constant stress accelerated life tests terminated by type-II censoring at one of the stress levels. J. Statist. Plann. Inference 2008, 138, 768-786. [CrossRef]

8. Abd El-Raheem, A.M. Optimal plans and estimation of constant-stress accelerated life tests for the extension of the exponential distribution under type-I censoring. J. Test. Eval. 2019, 47, 3781-3821. [CrossRef]

9. Abd El-Raheem, A.M. Optimal design of multiple accelerated life testing for generalized half-normal distribution under type-I censoring. J. Comput. Appl. Math. 2020, 368, 112539. [CrossRef]

10. Bai, D.S.; Kim, M.S.; Lee, S.H. Optimum simple step-stress accelerated life tests with censoring. IEEE Trans. Reliab. 1989, 38, 528-532. [CrossRef]

11. Gouno, E.; Sen, A.; Balakrishnan, N. Optimal step-stress test under progressive type-I censoring. IEEE Trans. Reliab. 2004, 53, 388-393. [CrossRef]

12. Miller, R.; Nelson, W. Optimum simple step-stress plans for accelerated life testing. IEEE Trans. Reliab. 1983, 32, 59-65. [CrossRef]

13. Balakrishnan, N.; Kundu, D.; Ng, H.K.T.; Kannan, N. Point and interval estimation for a simple step-stress model with type-II censoring. J. Qual. Technol. 2007, 39, 35-47. [CrossRef]

14. Mohie El-Din, M.M.; Abu-Youssef, S.E.; Ali, N.S.A.; Abd El-Raheem, A.M. Estimation in step-stress accelerated life tests for Weibull distribution with progressive first-failure censoring. J. Stat. Appl. Prob. 2015, 3, 403-411.

15. Mohie El-Din, M.M.; Abu-Youssef, S.E.; Ali, N.S.A.; Abd El-Raheem, A.M. Estimation in step-stress accelerated life tests for power generalized Weibull distribution with progressive censoring. Adv. Stat. 2015, 2015, 1-13. [CrossRef] [PubMed]

16. Mohie El-Din, M.M.; Abu-Youssef, S.E.; Ali, N.S.A.; Abd El-Raheem, A.M. Parametric inference on step-stress accelerated life testing for the extension of exponential distribution under progressive type-II censoring. Commun. Stat. Appl. Methods 2016, 23, 269-285.

17. Mohie El-Din, M.M.; Amein, M.M.; Abd El-Raheem, A.M.; Hafez, E.H.; Riad, F.H. Bayesian inference on progressive-stress accelerated life testing for the exponentiated Weibull distribution under Pprogressive type-II censoring. J. Stat. Appl. Pro. Lett. 2020, 7, 109-126.

18. Yin, X.K.; Sheng, B.Z. Some aspects of accelerated life testing by progressive stress. IEEE Trans. Reliab. 1987, $R-36,150-155$. [CrossRef]

19. Mohie El-Din, M.M.; Abu-Youssef, S.E.; Ali, N.S.; Abd El-Raheem, A.M. Classical and Bayesian inference on progressive-stress accelerated life testing for the extension of the exponential distribution under progressive type-II censoring. Qual. Reliab. Eng. Int. 2017, 33, 2483-2496. [CrossRef]

20. Bai, D.S.; Cha, M.S.; Chung, S.W. Optimum simple ramp-tests for the Weibull distribution and type-I censoring. IEEE Trans. Reliab. 1992, 41, 407-413. [CrossRef]

21. Ronghua, W.; Heliang, F. Statistical inference of Weibull distribution for tampered failure rate model in progressive stress accelerated life testing. J. Syst. Sci. Complex. 2004, 17, 237-243.

22. Abdel-Hamid, A.H.; AL-Hussaini, E.K. Progressive stress accelerated life tests under finite mixture models. Metrika 2007, 66, 213-231. [CrossRef] 
23. Abdel-Hamid, A.H.; AL-Hussaini, E.K. Inference for a progressive stress model from Weibull distribution under progressive type-II censoring. J. Comput. Appl. Math. 2011, 235, 5259-5271. [CrossRef]

24. AL-Hussaini, E.K.; Abdel-Hamid, A.H.; Hashem, A.F. One-sample Bayesian prediction intervals based on progressively type-II censored data from the half-logistic distribution under progressive stress model. Metrika 2015, 78, 771-783. [CrossRef]

25. Abdel-Hamid, A.H.; Abushal, T.A. Inference on progressive-stress model for the exponentiated exponential distribution under type-II progressive hybrid censoring. J. Stat. Comput. Simul. 2015, 85, 1165-1186. [CrossRef]

26. Abd El-Raheem, A.M. Estimation and optimal plans of multiple ramp-stress accelerated life tests for the generalized half-normal distribution. J. Egypt Math. Soc. 2018, 26, 347-364. [CrossRef]

27. Balakrishnan, N.; Aggarwala, R. Progressive Censoring: Theory, Methods, and Applications; Birkhäuser: Boston, MA, USA, 2000.

28. Balakrishnan, N.; Cramer, E. The Art of Progressive Censoring: Applications to Reliability and Quality; Birkhäuser: New York, NY, USA, 2014.

29. Arnold, B.C. Pareto Distributions; International Cooperative Publishing House: Fairland, MD, USA, 1983.

30. Serfling, R.J. Approximation Theorems of Mathematical Statistics; John Wiley \& Sons: Hoboken, NJ, USA, 1980.

31. Kloek, T.; Van Dijk, H.K. Bayesian estimates of equation system parameters: An application of integration by Monte Carlo. Econom. J. Eco. Soc. 1978, 46, 1-19. [CrossRef]

32. Zellner, A.; Rossi, P.E. Bayesian analysis of dichotomous quantal response models. J. Econom. 1984, 25, 365-393. [CrossRef]

33. Lindley, D.V. Approximate bayesian methods. Trabajos de Estadistica Y de Investigacion Operativa 1980, 31, 223-245. [CrossRef]

34. El-Din, M.M.; Abdel-Aty, Y.; Abu-Moussa, M.H. Statistical inference for the Gompertz distribution based on Type-II progressively hybrid censored data. Commun. Stat. Simul. Comput 2017, 46, 6242-6260. [CrossRef]

35. Mohie El-Din, M.M.; Nagy, M.; Abu-Moussa, M.H. Estimation and Prediction for Gompertz Distribution Under the Generalized Progressive Hybrid Censored Data. Ann. Data Sci. 2019, 6, 673-705. [CrossRef]

36. Mohie El-Din, M.M.; Amein, M.M.; El-Attar, H.E.; Hafez, E.H. Symmetric and Asymmetric Bayesian Estimation For Lindley Distribution Based on Progressive First Failure Censored Data. Math. Sci. Lett. 2017, 6, 255-261. [CrossRef]

37. Riad, F.H.; Hafez, E.H. Point and Interval Estimation for Frechet Distribution Based on Progressive First Failure Censored Data. J. Stat. Appl. Pro. 2020, 9, 181-191.

38. Abu-Moussa, M.H.; El-Din, M.M.M. On Estimation and Prediction for the Inverted Kumaraswamy Distribution Based on General Progressive Censored Samples. Pakistan J. Stat. Oper. Res. 2018, 717-736. [CrossRef]

39. Tierney, L.; Kadane, J.B. Accurate approximations for posterior moments and marginal densities. J. Am. Statist. Assoc. 1986, 81, 82-86. [CrossRef]

40. Miller, R. Survival Analysis; Wiley: New York, NY, USA, 1981.

41. Efron, B.; Tibshirani, R. Bootstrap methods for standard errors, confidence intervals, and other measures of statistical accuracy. Stat. Sci. 1986, 1, 54-75. [CrossRef]

42. Balakrishnan, N.; Sandhu, R.A. A simple simulation algorithm for generating progressively type-II censored samples. Am. Stat. 1995, 49, 229-230.

43. Zhu, Y. Optimal Design and Equivalency of Accelerated Life Testing Plans. Ph.D. Thesis, Rutgers University, Camden, NJ, USA, 2010.

Publisher's Note: MDPI stays neutral with regard to jurisdictional claims in published maps and institutional affiliations.

(C) 2020 by the authors. Licensee MDPI, Basel, Switzerland. This article is an open access article distributed under the terms and conditions of the Creative Commons Attribution (CC BY) license (http://creativecommons.org/licenses/by/4.0/). 